\title{
Structural Defects in Graphene
}

\author{
Florian Banhart, ${ }^{\mathrm{t}, *}$ Jani Kotakoski, ${ }^{ \pm, *}$ and Arkady V. Krasheninnikov ${ }^{ \pm, \xi_{, *}}$ \\ ${ }^{\dagger}$ Institut de Physique et Chimie des Matériaux, UMR 7504 CNRS, Université de Strasbourg, 23 rue du Loess, 67034 Strasbourg, France, ${ }^{\ddagger}$ Department of Physics, University of

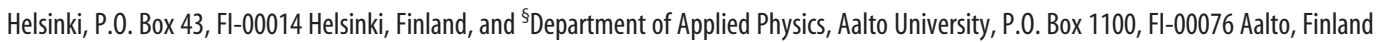

\begin{abstract}
Graphene is one of the most promising materials in nanotechnology. The electronic and mechanical properties of graphene samples with high perfection of the atomic lattice are outstanding, but structural defects, which may appear during growth or processing, deteriorate the performance of graphenebased devices. However, deviations from perfection can be useful in some applications, as they make it possible to tailor the local properties of graphene and to achieve new functionalities. In this article, the present knowledge about point and line defects in graphene are reviewed. Particular emphasis is put on the unique ability of graphene to reconstruct its lattice around intrinsic defects, leading to interesting effects and potential applications. Extrinsic defects such as foreign atoms which are of equally high importance for designing graphene-based devices with dedicated properties are also discussed.
\end{abstract}

KEYWORDS: carbon - graphene - defects - vacancies - interstitials - point defects · line defects · lattice reconstruction - electronic properties

*Address correspondence to banhart@ipcms.u-strasbg.fr, jani.kotakoski@iki.fi, akrashen@acclab.helsinki.fi.

Published online November 23, 2010. $10.1021 / \mathrm{nn} 102598 \mathrm{~m}$

C 2011 American Chemical Society
I $t$ is the second law of thermodynamics that dictates the presence of a certain amount of disorder in crystalline materials. But it is also due to the imperfection of material production processes that impurities and defects are always present in crystals. Such lattice imperfections have a strong influence on the electronic, optical, thermal, and mechanical properties of the solid. In fact, many of the characteristics of technologically important materials such as the conductance of semiconductors or the mechanical strength and ductility of metals are governed by defects. ${ }^{1}$

Defects in bulk crystals have been studied extensively for many decades. Twodimensional crystals, however, have been considered only recently. In fact, it was believed for a long time that they would be structurally unstable because of longwavelength fluctuations according to the Mermin-Wagner theorem. ${ }^{2}$ The situation changed, however, when single-layers of graphene were isolated for the first time by mechanical exfoliation. ${ }^{3}$ Graphene consists of a hexagonal monolayer network of $\mathrm{sp}^{2}$ hybridized carbon atoms. Graphene and its structural counterpart, hexagonal boronnitride, are the only two-dimensional crystalline materials we know today. ${ }^{4-6}$ The properties of graphene were expected to be outstanding, based on calculations addressing graphene as the parent material for carbon nanotubes. Therefore, the availability of graphene for experiments initiated a massive body of research, especially after large-scale synthesis methods like chemical vapor-deposition 7,8 and epitaxial growth ${ }^{9,10}$ on metal and SiC substrates were developed. Indeed, the predicted extraordinary properties have now been confirmed in many studies. ${ }^{4}$ Some of these properties can only be observed at an extremely low defect concentration, which, as we discuss later, is possible because of the high formation energies of point defects in graphene. Nevertheless, like in any other real material, structural defects do exist in graphene and can dramatically alter its properties. Defects can also be deliberately introduced into this material, for example, by irradiation or chemical treatments.

At first glance, what is true for a threedimensional material seems to be valid for graphene as well. The scattering of electron waves at defects has an enormous influence on the electrical conductivity. Dopant atoms change the local electronic structure or inject charge into the electron system of $\mathrm{sp}^{2}$-bonded carbon materials. Weaker bonds around defects affect the thermal conductivity and reduce the mechanical strength. However, graphene remains unique nevertheless; besides being truly two-dimensional, it can host lattice defects in reconstructed atom arrangements that do not occur in any other material. This is partly due to different possible hybridizations of carbon that allow different numbers 
of nearest neighbors and lead to the occurrence of different stable structures (carbyne, graphite, and diamond). Perhaps even more importantly, $\mathrm{sp}^{2}$-hybridized carbon atoms arrange themselves into a variety of different polygons, not only hexagons, to form different structures. The nonhexagonal rings may either introduce curvature in the sheet or leave it flat when the arrangement of polygons satisfies certain symmetry rules. This property does not appear in other bulk crystals, for example, semiconductors such as silicon. The reconstructions in the atomic network permit a coherent defective lattice without under-coordinated atoms. Although they have no dangling bonds, these reconstructed defects locally increase the reactivity of the structure and allow adsorption of other atoms on graphene.

Pure and structurally perfect graphene has shown outstanding electronic phenomena such as ballistic electron propagation with extremely high carrier mobilities $\left(10^{4} \mathrm{~cm}^{2} \mathrm{~V}^{-1} \mathrm{~s}^{-1}\right.$ at room temperature $\left.{ }^{3}\right)$ or the quantum Hall effect at room temperature. ${ }^{11}$ However, the absence of a bandgap in perfect graphene does not allow switching of graphene-based transistors with a high enough on-off ratio. Hence, graphene has to be modified even when making basic devices and, even more, for manufacturing sophisticated circuits.

Several experimental studies have shown the occurrence of either native or physically introduced defects in graphene. Transmission electron microscopy $\left(\right.$ TEM) ${ }^{12-16}$ and scanning tunneling microscopy $(\mathrm{STM})^{17,18}$ have been used to obtain images of defective graphene with atomic resolution. The interpretation of the experimental results was simplified by the fact that the theory of defects in graphene had already been developed to some extent in the context of carbon nanotubes ${ }^{19-23}$ and graphite. ${ }^{24-26}$ Carbon nanotubes consist of cylindrically closed graphene layers, and the behavior of defects in them was studied using graphene as the limiting zero-curvature case. However, the curvature in smaller nanotubes and the high aspect ratio of the tubes considerably affect the atomic structure and properties of defects, so that not all results obtained for tubes are directly applicable to graphene.

In this short review we present a survey of the hitherto reported structural defects in graphene. We will discuss the formation of defects and their influence on the properties of graphene. As will be clear from the following discussion, an essentially infinite number of various lattice defects can exist but we consider only the simplest ones. We will particularly focus on defects in single-layer graphene because defects typical for bi- and multilayer graphene also exist in graphite and have been discussed long before the graphene era. ${ }^{27-29}$

\section{DEFECT TYPES}

Defects in three-dimensional crystals are referred to as intrinsic when the crystalline order is perturbed without the presence of foreign atoms. The latter are denoted as impurities and constitute extrinsic defects. In macroscopic crystalline materials, intrinsic defects have different dimensionalities. Point defects, typically vacancies or interstitial atoms, are zero-dimensional, whereas the concept of dislocations is based on onedimensional lines of defects. Grain boundaries or stacking faults extend in two dimensions, while inclusions and voids have a finite size in all three dimensions. Foreign atoms may exist as zero-dimensional defects when they substitute individual atoms of the crystal or are located on interstitial sites. On the other hand, agglomerations of foreign atoms can extend to more dimensions. The reduced dimensionality of graphene itself decreases the number of possible defect types. Therefore, in graphene, the concept of zero-dimensional point defects is quite similar to bulk crystals, but line defects already play a different role. Truly threedimensional defects do not even exist in graphene.

It is well-known that defects are not always stationary and that their migration can have an important influence on the properties of a defective crystal. In graphene, each defect has a certain mobility parallel to the graphene plane. The mobility might be immeasurably low, for example, for extended vacancy complexes, or very high, for ex-
VOCABULARY: Mermin-Wagner theorem symmetries in zero-, one-, or twodimensional systems cannot be spontaneously broken at finite temperatures - Jahn-Teller distortion - nonlinear atomic arrangements having a degenerate electronic ground state undergo a geometric distortion to remove the degeneracy and thus lower the total energy of the system - Stone-Wales defect - generated by a pure reconstruction of a graphenic lattice (switching between pentagons, hexagons, and heptagons). No atoms are added or removed - defect reconstruction when an atom is removed from its lattice position, the lattice may relax into a lower energy state by changing the bonding geometry around the vacancy. In graphene, this leads to nonhexagonal bonding geometries - displacement threshold minimum energy that has to be transferred to an atom by a ballistic knock-on event so that the atom leaves its lattice site without recombination with the vacancy ample, for adatoms on an unperturbed graphene lattice. The migration is generally governed by an activation barrier which depends on the defect type and therefore increases exponentially with temperature.

Point Defects. Stone-Wales defect. As mentioned above, one of the unique properties of the graphene lattice is its ability to reconstruct by forming nonhexagonal rings. The simplest example is the Stone-Wales (SW) defect, ${ }^{30}$ which does not involve any removed or added atoms. Four hexagons are transformed into two pentagons and two heptagons [SW(55-77) defect] by rotating one of the $\mathrm{C}-\mathrm{C}$ bonds by $90^{\circ}$, as shown in Figure 1 . The SW(55-77) defect has a formation energy $E_{\mathrm{f}} \approx 5$ $\mathrm{eV}^{31,32}$ When the transformation occurs via an in-plane bond rotation by simultaneous movement of the two involved atoms, the kinetic barrier is ca. $10 \mathrm{eV} .{ }^{31}$ The defected structure retains the same number of atoms as 

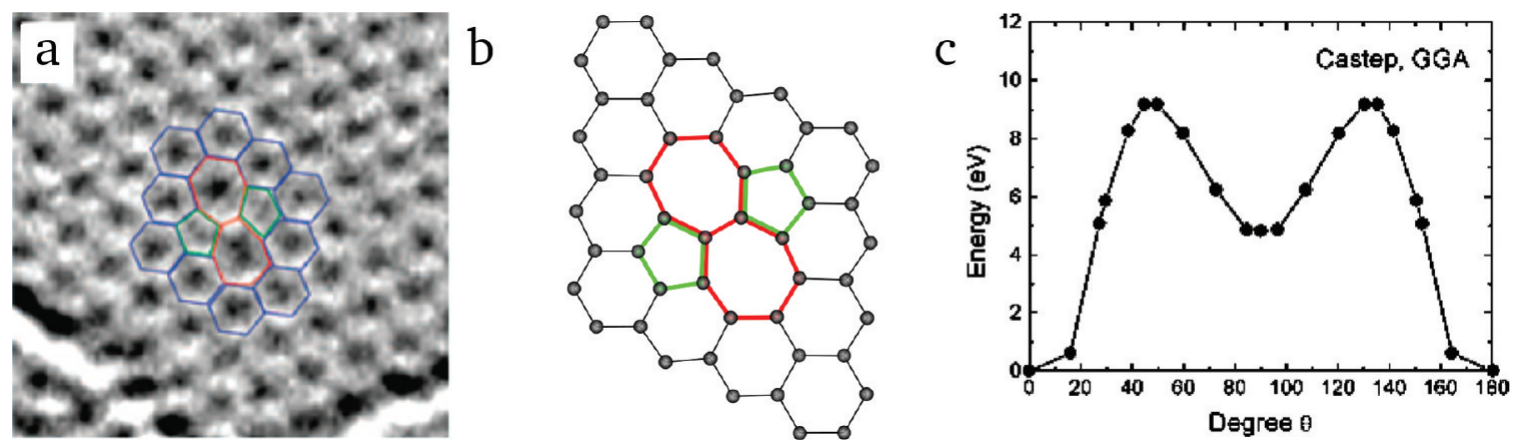

Figure 1. Stone-Wales defect SW(55-77), formed by rotating a carbon-carbon bond by $90^{\circ}$. (a) Experimental TEM image of the defect (courtesy of J. Meyer, reprinted from ref 14. Copyright 2008 American Chemical Society); (b) its atomic structure as obtained from our DFT calculations; (c) calculated energy barrier for bond rotation (courtesy of J. Robertson, reprinted with permission from ref 31. Copyright 2005 American Physical Society).

pristine graphene, and no dangling bonds are introduced. The reverse transformation has an energy barrier of ca. $5 \mathrm{eV}$. The high formation energy of the SW defect indicates a negligible equilibrium concentration, at least at typical experimental temperatures below $1000^{\circ} \mathrm{C}$. However, once the defect is formed under nonequilibrium conditions (e.g., rapid quenching from high temperature or under irradiation), the $5 \mathrm{eV}$ barrier for the reverse transformation should warrant its stability at room temperature. The SW defect shown in the TEM image (Figure 1a) may have appeared due to an electron impact. Such transformations are possible even if the electron beam transfers less than the threshold energy $T_{\mathrm{d}}$ to carbon atoms for their displacements because bond rotation requires less energy than a knock-on displacement ( $T_{\mathrm{d}}$ is the minimum energy which has to be transferred to a carbon atom to leave its lattice position without immediate recombination with the vacancy). Theoretical first-principles estimates of $T_{\mathrm{d}}$ have given ca. $18-22 \mathrm{eV}^{33,34}$ whereas experiments have yielded $18-20 \mathrm{eV} .^{35,36}$ Displacement thresholds of $18-20 \mathrm{eV}$ need electron energies of roughly $90-100 \mathrm{keV}$ as estimated within the McKinley-Feshbach approximation. ${ }^{35,37}$

Single Vacancies. The simplest defect in any material is the missing lattice atom. Single vacancies (SV) in graphene (or in the outermost layer of graphite) have been experimentally observed by TEM ${ }^{13,14}$ and STM. ${ }^{17}$ As can be seen in Figure 2, the SV undergoes a
Jahn-Teller distortion which leads to the saturation of two of the three dangling bonds toward the missing atom. One dangling bond always remains owing to geometrical reasons. This leads to the formation of a five-membered and a nine-membered ring $\left[\mathrm{V}_{1}(5-9)\right.$ defect]. The SV appears as a protrusion in STM images (Figure $2 c$ ) due to an increase in the local density of states at the Fermi energy which is spatially localized on the dangling bonds. It is intuitively clear that the formation energy of such a defect is high because of the presence of an under-coordinated carbon atom. Indeed, calculations have given a value $E_{\mathrm{f}} \approx 7.5 \mathrm{eV}^{22,24}$ which is much higher than the vacancy formation energies in many other materials (e.g., $4.0 \mathrm{eV}$ in $\mathrm{Si}^{38}$ or less than 3 $\mathrm{eV}$ in most metals ${ }^{39}$ ).

The calculated migration barrier for a SV in graphene is about $1.3 \mathrm{eV} .^{22,24}$ This already allows a measurable migration slightly above room temperature (100-200 $\left.{ }^{\circ} \mathrm{C}\right)$. Although the migration of point defects in graphene and related materials has not been directly accessible to observation, the annealing and reconstruction behavior, which is strongly influenced by defect migration, can be studied experimentally. For example, it has been noticed that structures related to graphene (e.g., nanotubes) reconstruct in situ under electron irradiation above a temperature of approximately $200-300{ }^{\circ} \mathrm{C}^{40,41}$ so that the atomic network remains coherent. ${ }^{42}$ Irradiation with electrons or ions at room temperature gives rise to a continuous formation

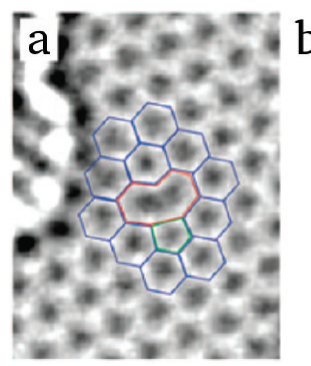

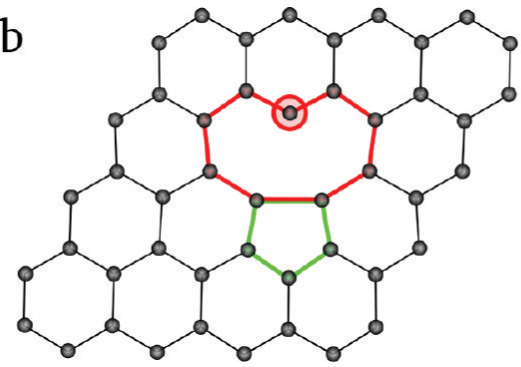

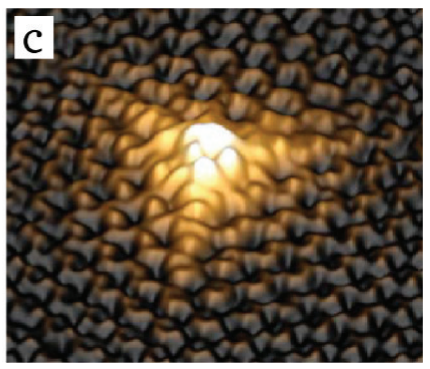

Figure 2. Single vacancy $\mathrm{V}_{1}(5-9)$ (a) as seen in an experimental TEM image (courtesy of J. Meyer, reprinted from ref 14. Copyright 2008 American Chemical Society); (b) its atomic structure obtained from our DFT calculations; (c) an experimental STM image of a single vacancy, appearing as a protrusion due to an increase in the local density of states at the dangling bond (marked with a circle in panel b) (courtesy of I. Brihuega, reprinted with permission from ref 17. Copyright 2010 American Physical Society). 

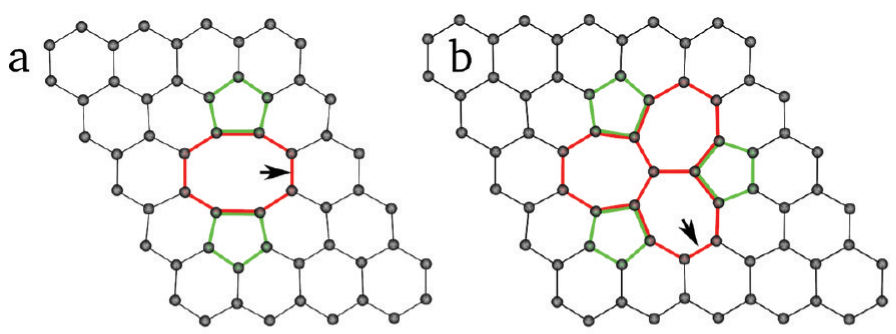

d

e
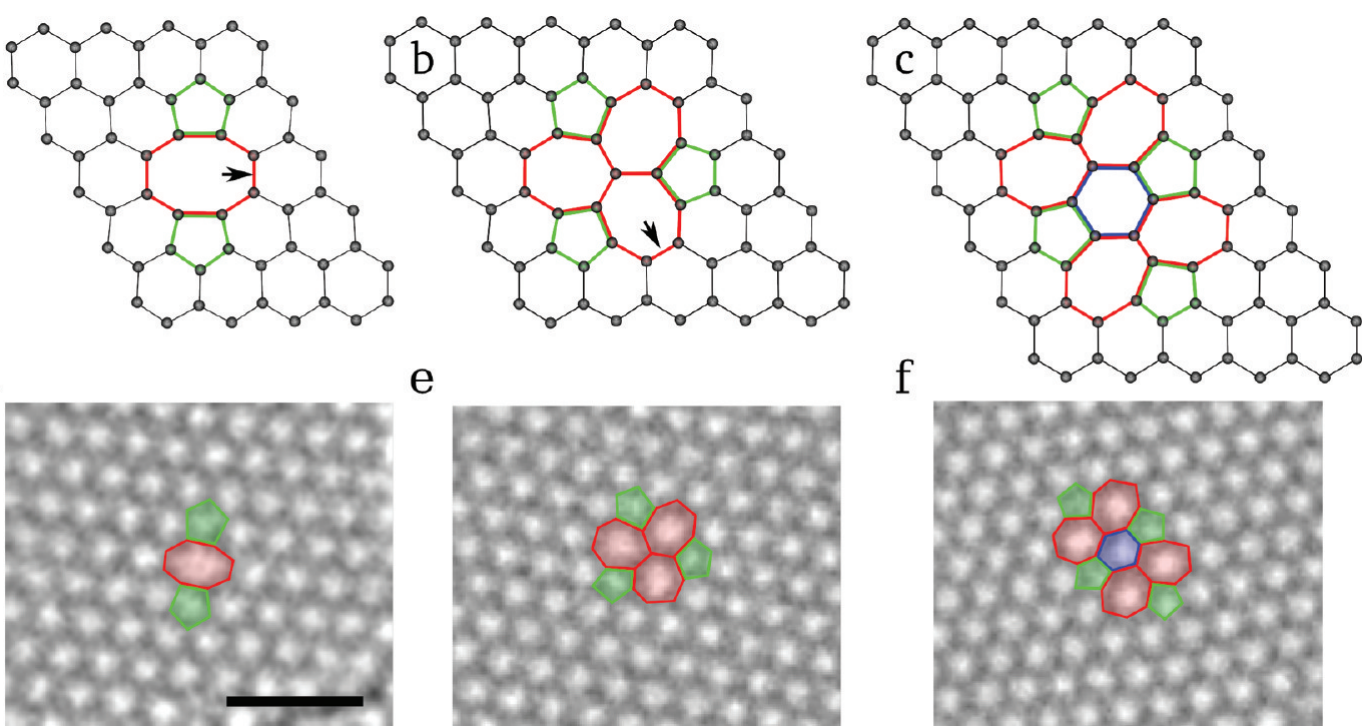

Figure 3. $(a-c)$ Atomic structures of reconstructed double vacancy defects in graphene as obtained from our DFT calculations; $(d-f)$ experimental TEM images of the same structures (courtesy of J. Meyer; J. Kotakoski et al., submitted for publication). (a,d) Double vacancy $V_{2}(5-8-5)$; $(b, e) V_{2}(555-777)$ transformed from the $V_{2}(5-8-5)$ defect by rotating a bond (marked in panel a); $(c, f) V_{2}(5555-6-7777)$ defect formed from $V_{2}(555-777)$ by another bond rotation (bond marked in panel b).

of defects, finally leading to the development of holes and amorphization. This only occurs when the electron energy is substantially above the displacement threshold for a carbon atom in the corresponding structure. ${ }^{36}$ Irradiation at electron energies close to the threshold gives rise to bond rotations, that is, reconstructions of the Stone-Wales type, even at room temperature (J. Kotakoski et al., submitted for publication).

Multiple Vacancies. Double vacancies (DV) can be created either by the coalescence of two SVs or by removing two neighboring atoms. As shown in Figure 3a, no dangling bond is present in a fully reconstructed DV so that two pentagons and one octagon $\left[\mathrm{V}_{2}(5-8-5)\right.$ defect] appear instead of four hexagons in perfect graphene. The atomic network remains coherent with minor perturbations in the bond lengths around the defect. Simulations ${ }^{22,24}$ indicate that the formation energy $E_{\mathrm{f}}$ of a DV is of the same order as for a SV (about $8 \mathrm{eV}$ ). As two atoms are now missing, the energy per missing atom (4 eV per atom) is much lower than for a SV. Hence, DVs are thermodynamically favored over SVs.

The $V_{2}(5-8-5)$ defect is not the only possible way for a graphene lattice to accommodate two missing atoms. In fact, it is not even the energetically favored one. Similar to the creation of a Stone-Wales defect, the rotation of one of the bonds in the octagon of the $V_{2}(5-$ 8-5) defect (Figure 3b) transforms the defect into an arrangement of three pentagons and three heptagons $V_{2}(555-777) .{ }^{43}$ The total formation energy of this defect is about $1 \mathrm{eV}$ lower than that of $\mathrm{V}_{2}(5-8-5)$. This defect is also frequently observed in electron microscopy experiments (J. Kotakoski et al., submitted for publication). One step further would be the transformation of the $V_{2}(555-777)$ into a $V_{2}(5555-6-7777)$ defect (Figure

www.acsnano.org 3c) by rotating another bond, which has experimentally been observed as well (J. Kotakoski et al., submitted for publication). The formation energy of this defect is between those of $V_{2}(5-8-5)$ and $V_{2}$ (555-777).

The migration of a DV needs an activation energy of ca. $7 \mathrm{eV}^{24}$ which is much higher than that for a SV (ca. $1.5 \mathrm{eV}$ ). This makes DVs in practice immobile up to very high temperatures at which DVs could migrate either by atom jumps ${ }^{24}$ or by switching between different reconstructions, e.g., $\mathrm{V}_{2}(5-8-5) \rightarrow \mathrm{V}_{2}(555-777) \rightarrow$ $\mathrm{V}_{2}$ (5-8-5) (J. Kotakoski et al., submitted for publication).

The removal of more than two atoms may lead to larger and more complex defect configurations. Generally, as an even number of missing atoms allows a full reconstruction (complete saturation of dangling bonds), such vacancies are energetically favored over structures with an odd number of missing atoms where an open bond remains. ${ }^{20}$ If a larger number of atoms is instantly removed from a small area (i.e., by an impact of an energetic ion), a reconstruction requires bending or warping of the layer because its surface area is considerably reduced. In such cases, the formation of a hole with unsaturated bonds around its circumference may be more likely. The formation of large holes has indeed been observed in electron microscopy experiments. ${ }^{16}$ Another way of reconstructing a highly defective graphene layer would be the (hitherto hypothetical) Haeckelite structure, ${ }^{44}$ which is a sheet consisting of pentagons and heptagons only. Yet another possibility would be an extended dislocation line, ${ }^{45}$ which would result from a linear arrangement of vacancies that close by saturating the dangling bonds over this line. However, both of the last two possibilities require a particular spatial arrangement of vacancies, and it is difficult to imagine how such structures could be cre- 
a

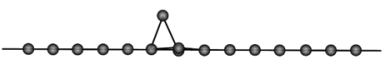

d

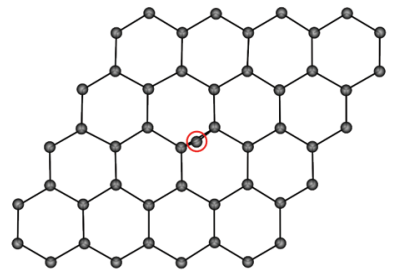

b

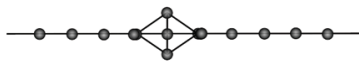

$\mathrm{e}$

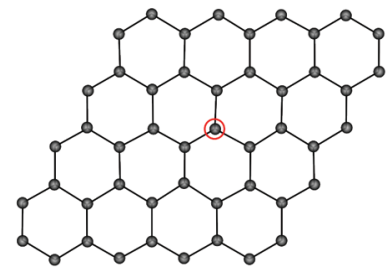

c

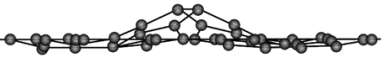

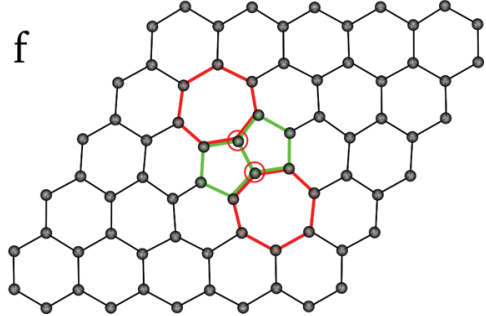

Figure 4. Carbon adatoms: ( $a, d)$ single adatom in the bridge; (b, e) single adatom in the dumbbell configuration; (c, $f$ ) the Inverse Stone-Wales defect $\mathrm{I}_{2}(7557)$ formed by two close adatoms as obtained from our DFT calculations.

ated in a physical or chemical process. Intuitively, one would expect to obtain a more-or-less random set of vacancies. Hence, it would seem more likely for the lattice to locally reconstruct around the vacancies and to form a correspondingly random set of nonhexagonal polygons. This is indeed what happens if the lattice is given enough time to relax before a large number of atoms are removed from a small area.

Carbon Adatoms. Interstitial atoms, as they appear in three-dimensional crystals, do not exist in graphene. This is because placing an atom to any in-plane position, for example, in the center of a hexagon, would require a prohibitively high energy. Rather than straining the local structure in two dimensions, additional atoms use the third dimension. The energetically favored position is the bridge configuration (on top of a carbon-carbon bond, as shown in Figure 4a). When a carbon atom interacts with a perfect graphene layer, the hybridization of the carbon atoms in the layer changes. Some degree of $\mathrm{sp}^{3}$-hybridization can appear locally so that two new covalent bonds can be formed between the adatom and the underlying atoms in the graphene plane. The binding energy of the carbon adatom is of the order of $1.5-2 \mathrm{eV}{ }^{46,47}$

In addition to the bridge position, other metastable configurations are possible. ${ }^{48}$ The small energy difference of about $0.3 \mathrm{eV}$ between the local and global minima indicates that adatoms migrate easily over the graphene surface. Indeed, calculations ${ }^{47}$ have given a migration barrier of about $0.4 \mathrm{eV}$. This means that carbon adatoms on flat graphene flakes migrate rapidly already at room temperature. Therefore, it should not be possible to detect them with TEM or STM, and some experimentally observed defect structures ${ }^{12}$ should be interpreted in terms of adatoms on defective graphene.

When a vacancy is created, the displaced atom may remain on the surface of the graphene layer. Since both adatoms and vacancies are relatively mobile, such a vacancy - "interstitial" pair (an analogue to a Frenkel pair) is unstable against recombination even below room temperature. However, as other possible reconstructions with the correct number of atoms exist (i.e., SW defects), it is not a priori clear whether the complete or partial recombination occurs. Not only migration on- the-lattice, but also through-the-lattice is possible for the adatoms by an exchange mechanism. To this end, the metastable dumbbell configuration (Figure 4b), which is higher in energy by $0.5 \mathrm{eV}^{49}$ than the bridge configuration, is important, especially in few-layer and multilayer graphene. The migration barrier for such a defect is about $0.9 \mathrm{eV}^{49}$ ).

When two migrating adatoms meet each other and form a dimer, they can be incorporated into the network of $\mathrm{sp}^{2}$-hybridized carbon atoms at the expense of local curvature of the network ${ }^{50}$ (Figure 4c). As compared to separated adatoms, the combined defect structure is energetically favored by approximately 2.6 $\mathrm{eV}$. The defect, composed of two pentagons and two heptagons $\left[I_{2}(7557)\right.$ defect $]$, was termed inverse Stone-Wales (ISW) defect. ${ }^{50}$ The arrangement of nonhexagonal rings is different from that in the SW defect. The total formation energy $E_{\mathrm{f}} \approx 5.8 \mathrm{eV}$ is even higher than for SW defects, so the concentration of such defects in otherwise flat carbon nanostructures should be negligible. However, they can appear under nonequilibrium conditions when two carbon atoms are displaced from their lattice positions and adsorbed on a graphenic structure so as to form a dimer. Such defects are immobile under ambient conditions, and when agglomerated, they may locally change the curvature of graphene flakes and even form hillocks. The possibility of tailoring the electronic properties of graphene by using such defects has been discussed, ${ }^{50,51}$ although the control over the precise location remains a challenge.

Foreign Adatoms. The effect of a foreign (noncarbon) atom on the properties of graphene depends on the bonding between the atom and graphene. ${ }^{52}$ If the bond is weak, only physisorption due to van der Waals interaction occurs. If the interaction is stronger, covalent bonding between the foreign atom and the nearest carbon atoms leads to chemisorption. Various bonding configurations, normally corresponding to highsymmetry positions such as on top of a carbon atom, on top of the center of a hexagon, or the bridge position, as discussed above, are possible.

A suitable way of studying the bonding between foreign atoms and graphene is to observe the migration 

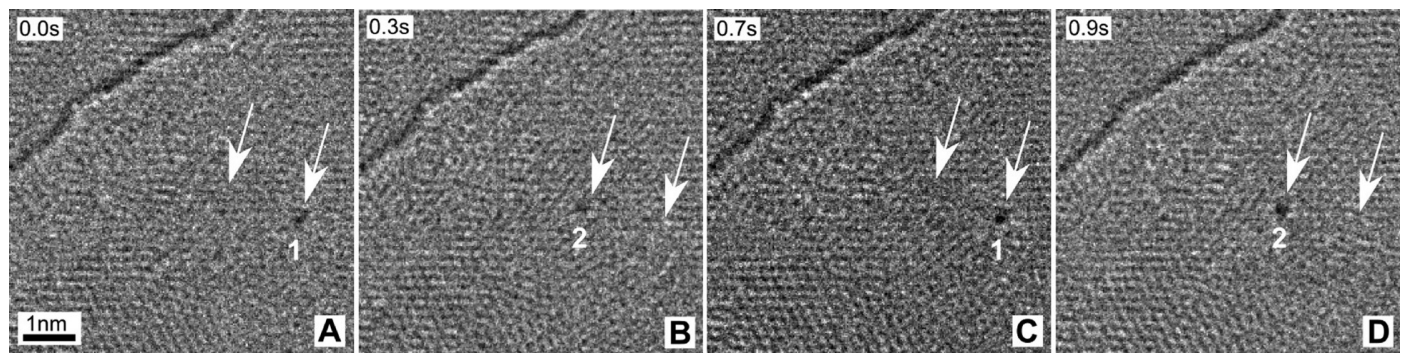

Figure 5. Jumping of a W atom (arrowed) on the surface of few-layer graphene at $480^{\circ} \mathrm{C}$ (in situ high-resolution TEM). The repeated jumping between two trapping sites ( 1 and 2 ) at a distance of $1.5 \mathrm{~nm}$ shows the attraction between a metal adatom and a reconstructed defect. ${ }^{55}$

of the atoms. ${ }^{53-55}$ However, only a few experimental studies about the migration of foreign species have been published to date. Most of the studies concentrated on the migration of impurity atoms on the surface of bulk graphite. For example, an activation energy of $0.28 \mathrm{eV}$ has been determined by in situ electron microscopy for the diffusion of Au on graphite, ${ }^{56} \mathrm{dem}$ onstrating the weak bonding between a perfect graphenic surface and metals. Theoretical studies have confirmed the weak bonding (activation energies $0.14-0.8 \mathrm{eV}$ ) between graphene and transition metal adatoms. ${ }^{57-61}$

In addition to positions on top of perfect graphene, adatoms can be pinned by structural defects which normally serve as reactive sites due to the locally increased reactivity of the $\pi$-electron system. For example, the $\mathrm{V}_{2}$ (555-777) defect can trap metal atoms ${ }^{55}$ by forming covalent bonds with them. The bonding energies are about $2 \mathrm{eV}$, allowing detrapping at high temperature or under particle irradiation. It has been found that the strain field around such a defect leads to an attractive interaction between the defect and a metal atom migrating on the surface over a scale of $1-2 \mathrm{~nm} .{ }^{55}$ This is shown in Figure 5 where a W atom jumps forth and back between two defective sites on graphene that both attract the $\mathrm{W}$ atom. A biaxial strain of $1 \%$ in the graphene lattice increases the adsorption energy for transition metal adatoms by $0.06-0.16 \mathrm{eV}$, depending on the metal species and the adsorption site. This may be of use for creating localization centers for metal atoms on graphene and for changing the electronic properties of graphene by charge injection from the attached metal atom.

It has been widely accepted that violations of the socalled "isolated pentagon rule" ${ }^{62,63}$ lead to particularly reactive sites in carbon structures. One example is the above-mentioned "inverse Stone-Wales defect"

$\mathrm{I}_{2}$ (7557) which appears when a carbon dimer occupies the open space over a hexagon and forms new bonds between the atoms on opposite sides, as shown in Figure 4c. The controlled introduction of defects such as $\mathrm{V}_{2}$ (555-777) or $\mathrm{I}_{2}$ (7557) significantly increases the reactivity of graphene, the inertness of which has caused many problems in controlling its properties.
Substitutional Impurities. Foreign atoms can also be incorporated into graphene as substitutional impurities. In this case, the impurity atom replaces one or two carbon atoms. Boron or nitrogen serve as the natural dopants in carbon structures since they have one electron less or more, respectively, but roughly the same atomic radius. Much larger atoms such as transition metal impurities have also received particular attention due to their ability to inject charge into the electron system of graphene. ${ }^{64}$ Owing to the different bond lengths between carbon and other atoms (in general longer than a carbon-carbon bond), most atoms are located slightly off the layer when occupying the substitutional position. ${ }^{60}$

Replacing carbon by boron or nitrogen atoms is of considerable interest because impurities not only move the position of the Fermi level but also change the electronic structure of graphene. ${ }^{65,66}$ As nitrogen doping has been shown to be an efficient way of introducing reactive sites into other carbon $\mathrm{sp}^{2}$ structures (and thus to functionalize these materials), ${ }^{67}$ one can expect a similar behavior for $\mathrm{N}$-doping of graphene, as simulations also indicate. ${ }^{66}$

Once created, substitutional dopants can be expected to be very stable due to strong covalent bonding. The migration of $\mathrm{Au}$ and $\mathrm{Pt}$ atoms, most likely on substitutional sites in graphene layers, has been the subject of an in situ study by electron microscopy. ${ }^{53}$ Activation energies of the order $2.5 \mathrm{eV}$ were obtained, but the diffusion might have been influenced by irradiation. ${ }^{68}$ Many transition metal (TM) atoms can form covalent bonds with under-coordinated $C$ atoms at a vacancy. ${ }^{60,69}$ The typical atomic configurations of (TM) - SV/DV complexes are shown in Figure 6. As the TM atomic radii are larger than those of the carbon atom, the metal atoms are displaced outward from the graphene plane. The binding energies $E_{\mathrm{b}}$ for the TMvacancy complexes are in the range of $2-8 \mathrm{eV}^{60,69}$ indicating strong bonding and pointing to a possible use of such structures in catalysis. Intriguing magnetic properties have been reported for many TM impurities. ${ }^{69}$ The strong bonding (e.g., 8.6 or $8.9 \mathrm{eV}$ for a tungsten atom trapped in a single or double vacancy, respectively, ${ }^{55}$ ) shows that such defect complexes are stable, 
a

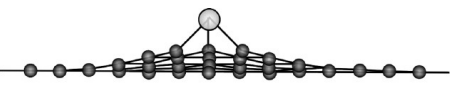

C

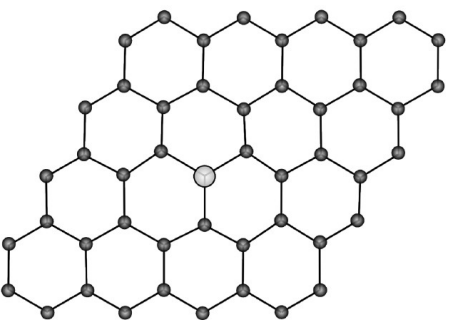

b

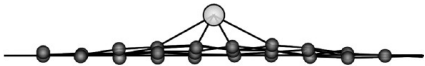

$\mathrm{d}$

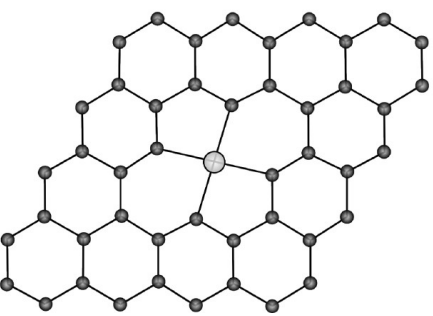

Figure 6. Typical atomic configurations of transition metal atoms adsorbed on single and double vacancies in a graphene sheet. Metal atom on a single vacancy: (a) side view; (c) top view. Note that the metal atom is $\sim 2 \AA$ off the surface. Metal atom on a double vacancy: (b) side view; (d) top view. The small dark circles denote carbon atoms; big lighter circles correspond to metal atoms.

and the metal atoms cannot be removed thermally or by irradiation with sub-MeV electrons.

Topology of Defective Graphene. Since the discovery of the fullerenes in the $1980 \mathrm{~s}^{70}$ it has been known that nonhexagonal rings induce local Gaussian curvature in a graphene sheet. Pentagons lead to positive (spherical) and heptagons to negative (saddle-like) curvature. Twelve pentagons lead to a complete spherical closure, for example, in the $\mathrm{C}_{60}$ molecule. In defective graphene, the arrangement of defects determines the local deviation from planarity. Defect domains might be created to induce hillocks or trenches in graphene. ${ }^{51}$ On the other hand, in symmetrical arrangements of pentagons and heptagons, like the $\mathrm{V}_{2}(555-777)$ defect and their more complex derivatives, positive and negative curvatures cancel each other, and the total Gaussian curvature of the structure remains zero, although small local deviations from ideally flat structure may be present in SW defects. ${ }^{32}$

One-Dimensional Defects. Dislocation-like Defects. Onedimensional defects have been observed in several experimental studies of graphene. ${ }^{12,71-73}$ Generally, these line defects are tilt boundaries separating two domains of different lattice orientations with the tilt axis normal to the plane. Such defects can be thought of as a line of reconstructed point defects with or without dangling bonds ${ }^{45,74,75}$ as shown in Figure 7. One example is a domain boundary which has been observed to appear due to lattice mismatch in graphene grown on a Ni surface. ${ }^{73}$ This defect consists of an alternating

line of pairs of pentagons separated by octagons (Figure 7c). Obviously, such a defect can be formed by aligning (5-8-5) divacancies along the zigzag lattice direction of graphene.

Some one-dimensional defects in graphene resemble the projection of a dislocation in a conventional crystal. However, the conventional concept requires a Burgers vector and a dislocation line, which can only appear in a three-dimensional crystal. ${ }^{1}$ Screw dislocations require a three-dimensional strain field and can therefore not exist in graphene. However, an equivalent of an edge dislocation can be imagined in graphene, but only in its projection onto a plane because no dislocation line normal to the layer exists. The concept of a dislocation in graphene has been described as a semi-infinite strip of width $b$ ( $b$ corresponds to the Burgers vector) introduced into the layer ${ }^{74}$ as shown in Figure 8 . The role of the dislocation core is played by a pentagon-heptagon pair which appears at the end of the strip and has no dangling bonds.

Line defects in graphene frequently separate domains of different crystal orientation. Several examples have been shown in the growth of graphene on metal surfaces. ${ }^{71,73}$ They arise because simultaneous nucleation of graphene at different points may lead to independent two-dimensional domains, corresponding to grains in three-dimensional crystals. Normally, metal surfaces of hexagonal symmetry [e.g., the (111) surface of cubic or the (0001) surface of hexagonal crystals] are used to grow graphene by chemical vapor deposition. The misfit between metal and graphene may lead to differing lattice orientations for different grains. Therefore, a line defect appears when two graphene grains with differing orientations coalesce.

The linear defect corresponding to grain boundaries in graphene should be of paramount importance. It is well-known that the properties of polycrystalline materials are often dominated by the size of their grains and by the atomic structure of the grain boundaries, but the role of such structures should be pronounced in two-dimensional materials such as graphene where even a line defect can divide and disrupt a crystal. In
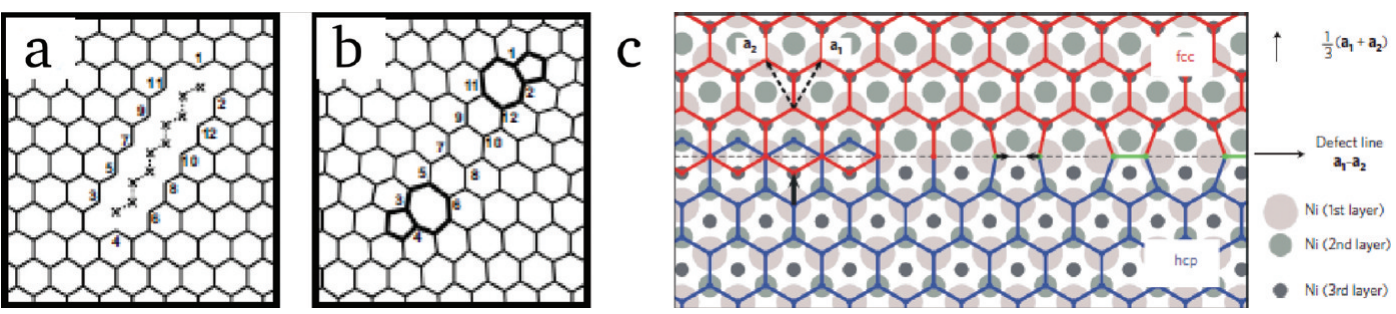

Figure 7. $(a, b)$ Line defect formation from aligned vacancy structures (courtesy of G.-D. Lee, reprinted with permission from ref 45. Copyright 2008 American Physical Society); (c) grain boundary defect structure consisting of pentagon-pairs and octagons in graphene grown on a Ni substrate (courtesy of M. Batzill, reprinted with permission from ref 73. Copyright $2010 \mathrm{Na}-$ ture Publishing Group). 


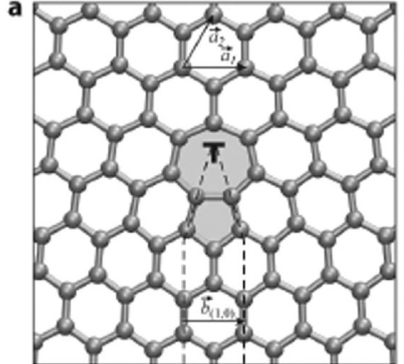

$(1,0)$ dislocation

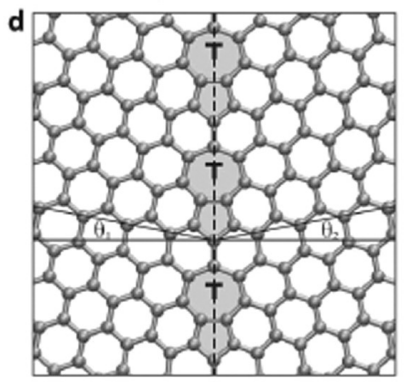

LAGB I

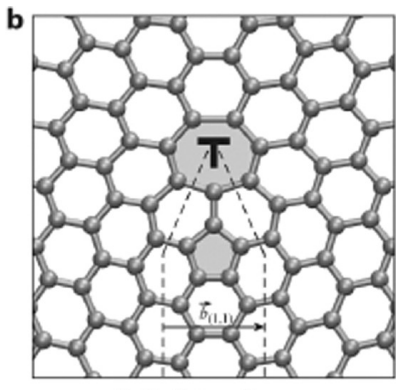

$(1,1)$ dislocation

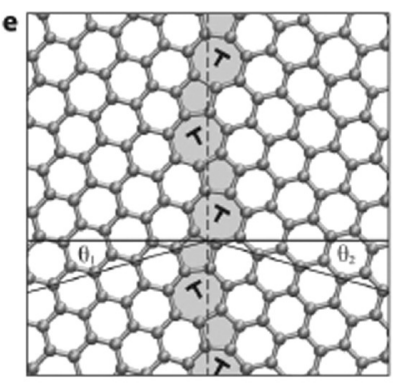

LAGB II

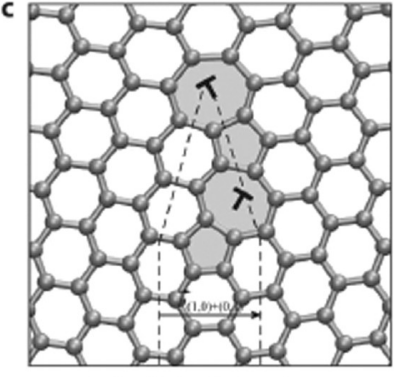

$(1,0)+(0,1)$ dislocation

f
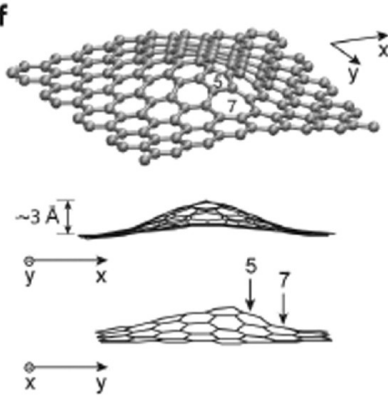

Figure 8. Dislocations in graphene (courtesy of O. Yazyev, reprinted with permission from ref 74. Copyright 2010 American Physical Society). (a-c) Atomic structures of $(1,0)$ and $(1,1)$ dislocations, and a $(1,0)+(0,1)$ dislocation pair, respectively; $(d, e)$ atomic structures of the $\theta=21.8^{\circ}$ large-angle grain boundary and the $\theta=32.2^{\circ}$ symmetric large-angle grain boundaries; (f) buckling of the graphene layer due the presence of a $(1,0)$ dislocation.

particular, grain boundaries may govern the electronic transport in such samples. ${ }^{74}$

Defects at the Edges of a Graphene Layer. Each graphene layer is terminated by edges with the edge atom being either free or passivated with hydrogen atoms. The simplest edge structures are the armchair and the zigzag orientation. They can reconstruct ${ }^{76,77}$ as shown in Figure 9. Also any other direction in between these two can be imagined. However, the zigzag and armchair orientations seem to be preferred, possibly because they minimize the number of dangling bonds at the edge. Defective edges can appear because of local changes in the reconstruction type or because of sustained removal of carbon atoms from the edges. This can be done by sputtering edge atoms ${ }^{16,78}$ with electrons having energies below the threshold energy for displacing atoms from perfect graphene (about $80 \mathrm{keV}^{36}$ ). Under these conditions, armchair edges can be transformed to zigzag edges. ${ }^{16}$ An intermediate structure can be considered as a defective edge. A simple example of an edge defect is the removal of one carbon atom from a zigzag edge. This leads to one pentagon in the middle of a row of hexagons at the edge. Other edge reconstructions result in different combinations of pentagons and heptagons at the edge as shown in Figure 9. Besides, hydrogen atoms and other chemical groups that can saturate dangling bonds at the edge under ambient conditions may be considered as disorder, dramatically increasing the number of possible edge defects.

Defects in Bilayer Graphene. Although defects in multilayer graphene will not be addressed at length in this review, some defect-related effects in bilayer graphene are important and will hence be briefly mentioned. Bi-

www.acsnano.org layer graphene consists of two stacked monolayers which may or may not be shifted with respect to each other. Similar to graphite, the interlayer distance in bilayer graphene is approximately $3.35 \AA$, as dictated by the weak van der Waals interaction between the layers. The electronic properties of a bilayer might be superior to monolayer graphene because a nonzero bandgap can be created. ${ }^{79,80}$ Although defects can exist in both layers independently, there is a tendency to form covalent interlayer bonds when adatoms are located between the layers. ${ }^{24,81,82}$ Thus, the creation of a Frenkel pair (adatom - vacancy complex) in one layer may lead to a locally changed hybridization in the neighboring layer so that opposite carbon atoms from the two layers get connected via one extra carbon atom.

Figure 10 shows some configurations of defects in bilayer graphene. ${ }^{81}$ Creating two vacancies in neighboring layers (interlayer double vacancy) leads to a bridging bond with a length (depending on the configuration) between 1.38 and $1.43 \AA$ (Figure 10a,b). Another possible bilayer defect is the "spiro interstitial", where the bridge atom is 4-fold coordinated (Figure 10c). Such defects appear to be important for controlling the morphology of graphene layers under particle irradiation and heat treatment, as they prevent the coalescence of adjacent layers. ${ }^{83}$ Other defects such as close metastable Frenkel pairs, sometimes referred to as "Wigner defects", exist in bilayer graphene. ${ }^{25,82}$

\section{GENERATION OF DEFECTS}

The high formation energy of a single vacancy in graphene (7.5 eV) does not allow any detectable concentration of point defects in thermal equilibrium at 
a

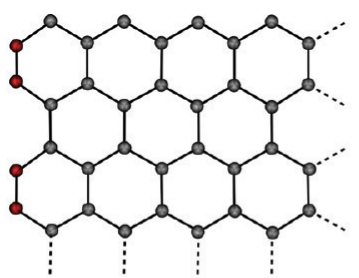

b

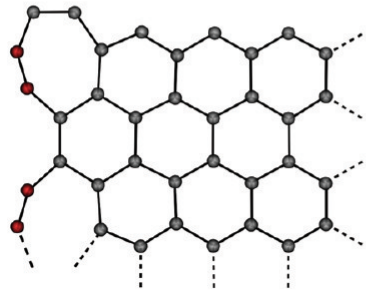

c

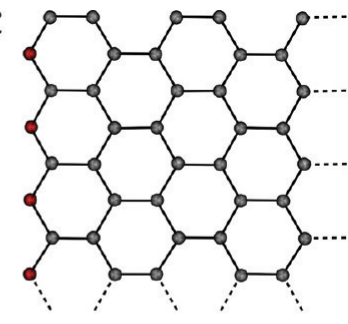

d

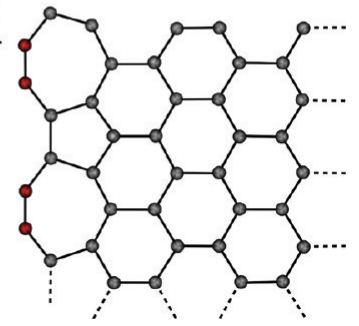

Figure 9. Different edge reconstructions in graphene: (a) armchair (AC) edge; (b) (677) reconstructed AC edge; (c) zigzag (ZZ) edge; (d) (57) reconstructed $Z Z$ edge. The edge atoms (shown in red) can be passivated with hydrogen. According to DFT calculations, ${ }^{76}$ the naked (57) ZZ edge has the lowest energy, while the AC edge is energetically favorable if all the dangling bonds are passivated.

temperatures below the melting resp. sublimation temperature of graphite $\left(3675^{\circ} \mathrm{C}\right)$. However, there are three mechanisms which can lead to nonequilibrium defects in graphene: (1) crystal growth; (2) irradiation with energetic particles, for example, electrons or ions; and (3) chemical treatment. Figure 11 shows an electron microscopy image as an example of a graphene layer where point and line defects from the growth process as well as radiation-induced defects are visible.

Crystal Growth. Since the large-scale growth of a graphene layer does normally not occur slowly atomby-atom from one nucleus but rather as a relaxation of a metal-carbon system with many nuclei, for example, in chemical vapor deposition (CVD), it is natural to expect defects in the as-grown material. Generally, high temperature growth facilitates the relaxation toward thermal equilibrium, and defects can anneal rapidly. However, defects are a well-known problem in lowtemperature growth. Because of the high formation energy of vacancies and fast migration of adatoms in graphene, it is unlikely that there are any isolated vacancies in graphene after growth. This has been confirmed by the high carrier mobility in CVD-grown graphene ${ }^{84}$ which would not be expected at a considerable density of vacancies.
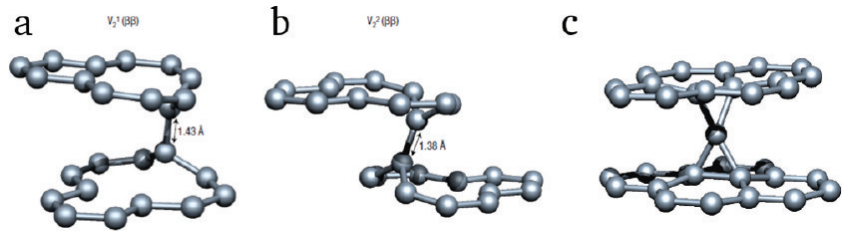

Figure 10. Interlayer double vacancy structures in bilayer graphene (courtesy of M. Heggie and C. Ewels, reprinted with permission from ref 81. Copyright 2003 Nature Publishing Group): (a) $\mathrm{V}_{2}{ }^{1}(\boldsymbol{\beta} \beta)$, (b) $\mathrm{V}_{2}{ }^{2}(\boldsymbol{\beta} \beta)$, and (c) spiro-interstitial (a four-coordinated carbon atom between the layers).

Line defects around two-dimensional domains can be generated when the nucleation of graphene layers occurs simultaneously at different locations on a substrate and coalescence of these domains. This corresponds to the formation of grain boundaries in the growth of conventional crystals. For graphene this has been observed on catalytically active metals, for example, on (111) surfaces of fcc crystals such as nickel. ${ }^{73}$ Since the adsorption energy of graphene on metals is low, different orientations of graphene relative to the metal surface are possible. For example, the epitaxial relationship between graphene and the (111) surface of an fcc metal allows an hcp as well as an fcc geometry. Coalescence of these two domains leads to the formation of a defect line, consisting of pentagons and octagons (such a grain boundary can also be constructed by aligning a row of divacancies in the zigzag direction). ${ }^{73}$

Particle Irradiation. Irradiation of graphene with electrons or ions can generate point defects due to the ballistic ejection of carbon atoms. ${ }^{35,85,86}$ As mentioned above, the threshold energy of approximately $18-20$ $\mathrm{eV}$ has to be transferred to a carbon atom to leave its lattice site. The atom can be sputtered away from graphene or get adsorbed on the sheet and migrate on its surface as an adatom. The effect of irradiation has been studied in detail in electron microscopy experiments $\mathrm{s}^{6,12,13}$ where irradiation and imaging can be done with the same electron beam, and the formation of defects is observable in situ at atomic resolution. Uniform irradiation of larger areas leads to a generation of randomly distributed vacancies. However, due to increased strain and/or under-coordinated atoms, the defective areas, for example, where a vacancy already exists, show an increased rate of defect formation. Reconstructions of vacancies and Stone-Wales transformations have been observed by in situ electron microscopy (J. Kotakoski et al., submitted for publication). ${ }^{6}$ Defects can also be generated in preselected positions with a highly focused electron beam or by using masking techniques. Modern electron microscopes with aberration-corrected condensers allow focusing an electron beam onto a spot of approximately $1 \AA$ in diameter thereby creating vacancies with almost atomic selectivity. ${ }^{87}$

Another physical method which has been used for defect production in graphene is ion irradiation. ${ }^{18,88-91}$ It can be used to selectively produce certain defects (typically vacancies) ${ }^{92}$ or to pattern and cut graphene with a precision down to $10 \mathrm{~nm}$ utilizing a focused ion beam (FIB). ${ }^{93}$ In Figure 12a,b, the number of SVs and DVs in graphene irradiated with different noble gas ions is shown as a function of the ion energy. It is evident that for suspended graphene the number of sputtered atoms is about one for nearly all noble gas ions. Graphene becomes essentially transparent for highenergy ions..$^{92}$ Figure $12 \mathrm{c}$ presents the displacement threshold in graphene (minimum energy for displacing 
a carbon atom with the atom velocity in a certain direction) as a function of the incidence angle. ${ }^{94}$ Figure $12 d$ shows the evolution of the Raman spectrum for graphene during continuous $\mathrm{Ar}^{+}$ion irradiation, demonstrating the appearance of the so-called D peak, associated with defects. ${ }^{88}$

Chemical Methods. The reactions of carbon atoms in a graphene layer with other species can lead to the loss of atoms and hence to defects. However, the high inertness of graphene (apart from edge positions that are highly reactive) only allows a very limited number of possible reactions at room temperature. Oxidation is the most common one, for example, in an oxidizing acid $\left(\mathrm{HNO}_{3}\right.$ or $\left.\mathrm{H}_{2} \mathrm{SO}_{4}\right)$. In such a treatment it is possible to attach oxygen and hydroxyl $(\mathrm{OH})$ or carboxyl $(\mathrm{COOH})$ groups to graphene. When graphene is covered more or less uniformly with hydroxyl or carboxyl groups, the material is called graphene oxide, which is essentially a highly defective graphene sheet functionalized with oxygen groups. ${ }^{95}$ Plasma treatment and adsorption of atomic hydrogen on a graphene surface followed by its self-organization and hydrogen island formation ${ }^{96}$ can also be referred to in the context of graphene treatment by chemical methods.

\section{PROPERTIES OF DEFECTIVE GRAPHENE}

Chemical Properties. It is intuitively clear that defects associated with dangling bonds should enhance the reactivity of graphene. Indeed, numerous simulations ${ }^{66,97}$ indicate that hydroxyl, carboxyl, or other groups can easily be attached to vacancy-type defects. The same is true for graphene edges that are normally saturated with hydrogen. Simulations also show that reconstructed defects without dangling bonds such as SW defects or reconstructed vacancies locally change the density of $\pi$-electrons ${ }^{66,98}$ and may also increase the local reactivity. ${ }^{99}$ Indeed, experiments provide evidence that the trapping of metal atoms occurs in reconstructed vacancies. ${ }^{55}$ Thus, the controlled creation of defects with a high spatial selectivity can be used for the local functionalization of graphene samples, the development of electrical contacts with metal electrodes, and for the creation of graphene ribbons with the designed properties by various chemical methods.

Electronic Properties. Defects strongly affect the electronic properties of graphene. From a theoretical point of view, the Dirac equation - which replaces the Schrödinger equation for electrons in graphene- has to be modified when defects are in the lattice. This will naturally have an influence on the electronic structure. The overlap of $\mathrm{p}_{z}$-orbitals determines the electronic properties but is altered in the vicinity of structural defects. First, bond lengths in the strain fields of defects are different from those in the perfect lattice. Furthermore, defects lead to a local rehybridization of sigma and piorbitals which again changes the electronic structure. A local curvature around defects (due to nonhexago-

www.acsnano.org

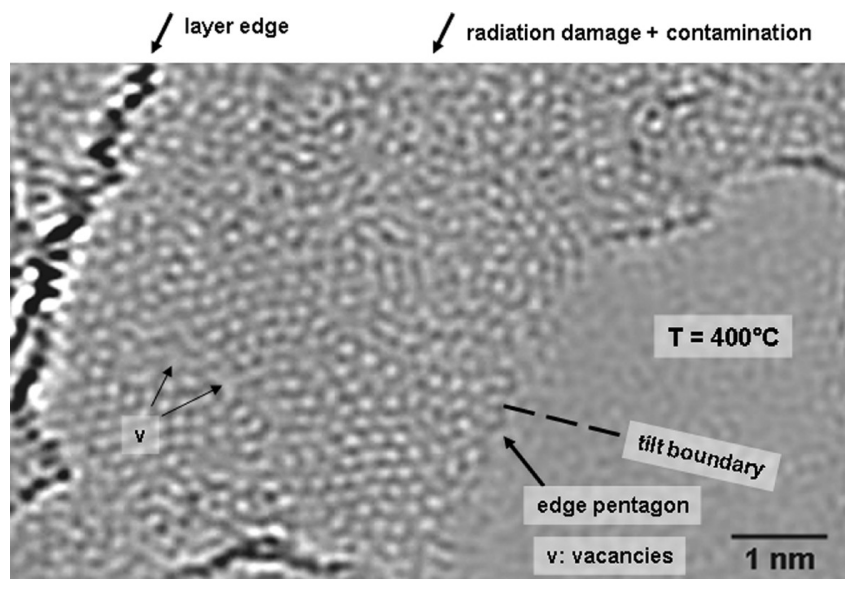

Figure 11. Scanning transmission electron microscopy image (bright field) of a single graphene layer with grown-in defects (e.g., the tilt boundary) and defects due to electron irradiation in the microscope (e.g., vacancies). Electron irradiation and imaging has been carried out at $400{ }^{\circ} \mathrm{C}$ and an electron energy of $200 \mathrm{keV}$ (STEM image by O. Cretu).

nal rings or adatom complexes created via chemical or physical treatment) also has an influence on the rehybridization. All defects lead to scattering of the electron waves and change the electron trajectories. ${ }^{100,101}$

Studies of the influence of structural defects on the electrical properties of graphene have just begun, ${ }^{101}$ and there is still a lack of reproducible experimental results, as compared to the vast amount of the available theoretical data. Therefore, the present survey is unavoidably incomplete, and just a snapshot of the present activities can be given. Besides, the theoretical aspects of the electronic properties of graphene with defects have been addressed in detail in a recent review. ${ }^{5}$ Thus we will only briefly summarize the influence of structural defects on the electronic structure and transport properties. We do not consider the effects originating from weakly attached organic ${ }^{102,103}$ or inorganic ${ }^{104}$ surface species on perfect graphene, because these are no structural defects, nor discuss the effects of ripples in free-standing or substrate-supported graphene.

Electronic Structure of Graphene in the Presence of Defects. The electronic structure of graphene with point and line defects has been studied in a considerable number of papers. ${ }^{5,17,73,101,105,106}$ Point defects give rise to localized states near the Fermi energy in $\mathrm{sp}^{2}$-bonded materials, leading to the protrusions that appear in STM images. ${ }^{17}$ Some vacancy-type ${ }^{107}$ and SW ${ }^{98}$ defects open a local bandgap (up to $0.3 \mathrm{eV}$ ) in graphene, which may be quite important for defect-mediated engineering of the local electronic structure. Some of these defects have already been generated by electron irradiation (J. Kotakoski et al., submitted for publication). The results of calculations, however, strongly depend on the technique of calculating the electronic structure, so that the predictions on gap opening should be experimentally verified (e.g., by measuring local density of states by STM) before moving forward along this av- 

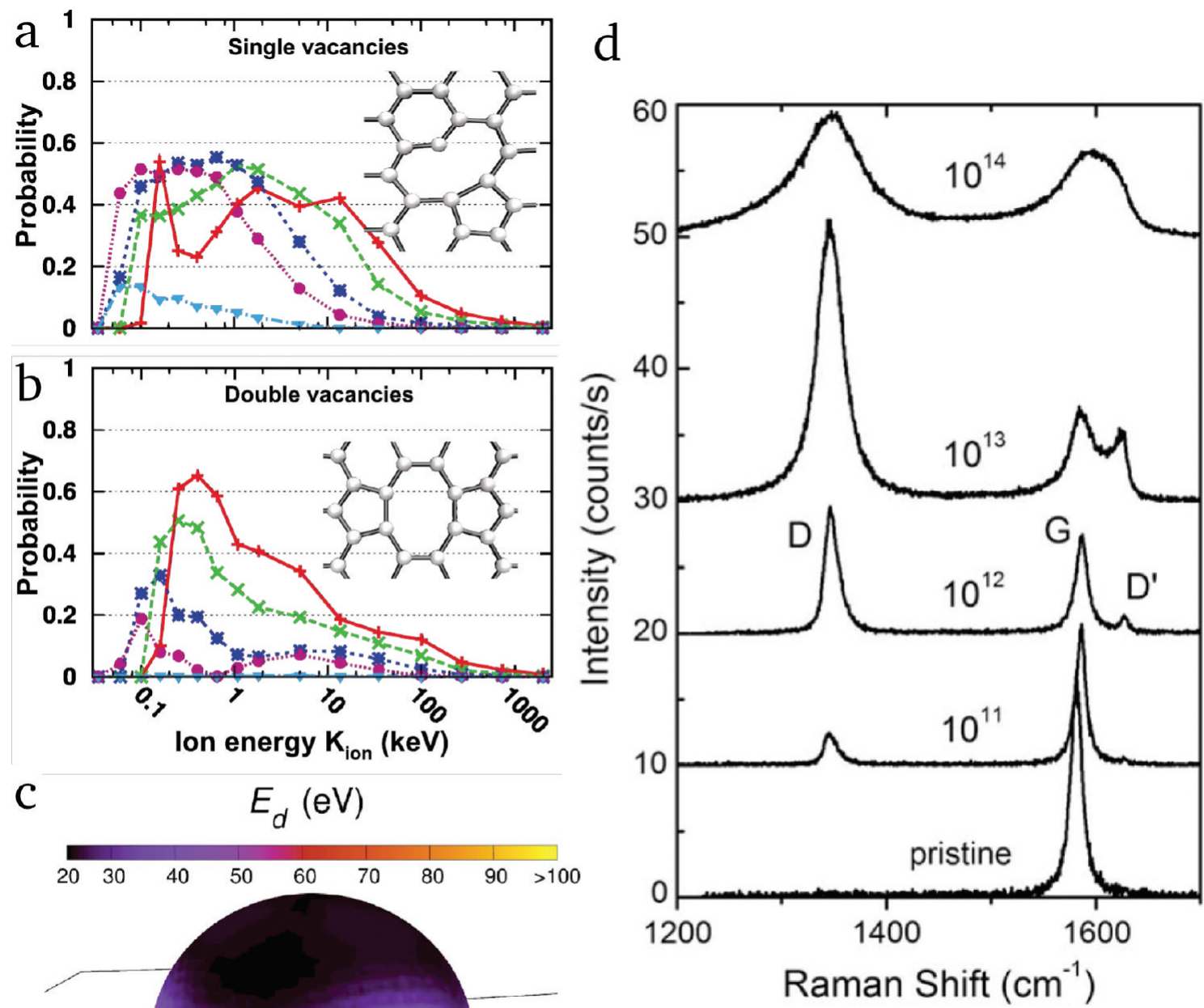

C $E_{d}(\mathrm{eV})$

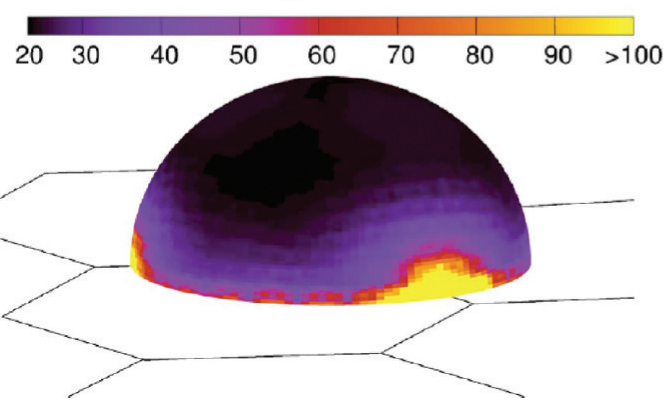

Raman Shift $\left(\mathrm{cm}^{-1}\right)$

Figure 12. $(a, b)$ Probability for creating a single or double vacancy, respectively, due to irradiation with different noble gas ions, as a function of the ion energy; ${ }^{92}$ (c) displacement threshold energy as a function of the space angle for a carbon atom in graphene (courtesy of C. Ewels, reprinted with permission from ref 94. Copyright 2007 American Physical Society); (d) ion irradiation effect on graphene as seen with Raman spectroscopy (reprinted with permission from ref 88. Copyright 2010 Elsevier). In panel $\mathbf{d}$ the evolution of the first-order Raman spectra of a monolayer graphene sample deposited on a SiO ${ }_{2}$ substrate is shown at different stages of $\mathrm{Ar}^{+}$ion bombardment using a $\lambda=514 \mathrm{~nm}$ laser. The ion doses are indicated next to the respective spectrum in units of $\mathrm{Ar}^{+} / \mathrm{cm}^{2}$.

enue for the development of graphene-based electronics. Stone-Wales defects in nanoribbons may already create new states in the gap. ${ }^{66}$ The position of the states can, in theory, be controlled by the location of the defect with regard to ribbon edges and the defect density.

Line defects composed of nonhexagonal rings ${ }^{73}$ should give rise to electronic states localized in the transverse direction and extended along the line. Such defects can enhance the conductivity along the line ${ }^{73}$ due to a larger number of conducting channels, which may be used for building blocks in atomic-scale allcarbon electronics. Of importance in real systems is the electronic transport through grain boundaries in polycrystalline graphene. These grain boundaries consist of periodic arrays of dislocations. Reflection or perfect transmission of charge carriers, depending on the structure of the grain boundary, has been predicted. ${ }^{106}$ The introduction of such arrays of dislocations could be used to control charge currents in devices.

Defects as Scattering Centers. Point defects in graphene act as scattering centers for electron waves. Thus, one can expect that such defects will result in a drop of conductance through graphene ribbons, although defects with/without dangling bonds and with different symmetry may affect the electronic transport in a different way. Besides, defects in the inner part of the ribbon and at the edges may have different effects. ${ }^{108}$ Numerous simulations showed that vacancies, SW defects, and adatoms including hydrogen and various ligands decrease the conductivity ${ }^{109-112}$ through the bulk conducting channels, while metallic edge states are more 
TABLE 1. Formation and Migration Energies of Point Defects in Graphene. The Values Are Taken from Theoretical Work

\begin{tabular}{|c|c|c|c|c|}
\hline defect type & configuration & formation energy [eV] & migration energy [eV] & references \\
\hline Stone-Wales & $55-77$ & $4.5-5.3$ & 10 & 31,32 \\
\hline single vacancy & $5-9$ & $7.3-7.5$ & $1.2-1.4$ & 24 \\
\hline \multirow[t]{3}{*}{ double vacancy } & $5-8-5$ & $7.2-7.9$ & 7 & 22,24 \\
\hline & $555-777$ & $6.4-7.5$ & 6 & 43,55 \\
\hline & $555-6-777$ & 7 & 6 & J. Kotakoski et al., submitted \\
\hline adatom $^{a}$ & & $6-7$ & 0.4 & 46 \\
\hline inverse SW & $57-57$ & $5.8^{b}$ & & 50 \\
\hline adatom -SV pair & & 14 & & 50 \\
\hline
\end{tabular}

${ }^{a}$ The formation energy of the adatom can be calculated as a difference between the atomization energy of graphene $(7.5-8 \mathrm{eV})$ and the adsorption energy $(1.4-2 \mathrm{eV})$. ${ }^{b}$ Note that two adatoms are involved.

robust under a variety of chemical environments. ${ }^{97}$ The effects of complicated defects, composed of nonhexagonal rings, on the conductance have not been studied so far, but one may expect that they are less pronounced as compared to those generated by defects with dangling bonds.

Charge Carrier Doping of Graphene by Defects. The doping of graphene to change its electronic properties has to be carried out by modifying the $\pi$-electron system. Such a doping can be done in two ways. The first is "selfdoping" where intrinsic defects modify the band structure locally. The other way would be doping in the conventional way, that is, by adding foreign atoms to graphene that inject charge into the conduction or valence band.

Self-doping ${ }^{5}$ by intrinsic defects is due to a slight electron-hole asymmetry in perfect graphene. ${ }^{113}$ This causes a change of the Fermi energy of point defects and leads to an injection of charge into the whole electron system. Line defects may have the same effect on a larger scale. Self-doping has experimentally been successful for line defects such as rows of pentagons and octagons that have been suggested to act as conducting metallic wires. ${ }^{73}$

Doping by foreign species has already been done in several ways. An injection of charge into the electron system of graphene has been achieved from metal contacts ${ }^{114}$ or by attaching organic molecules on a perfect graphene layer. ${ }^{102,115}$ However, the adsorption of organic species is weak and desorption starts at temperatures below $100{ }^{\circ} \mathrm{C} .{ }^{116}$ Nevertheless, the localization of dopants in graphene could be achieved on defects where an increased reactivity prevails. Foreign atoms on substitutional sites appear to be unfavorable because the strong scattering of the conduction electrons at such atoms might deteriorate the electronic properties of graphene. ${ }^{5,117}$ Reconstructed defects, however, could be more attractive because the coherence of the graphene lattice is preserved and the foreign dopant atoms are nevertheless firmly attached. ${ }^{55}$

Doping by substitutional impurities is quite straightforward if $\mathrm{B}$ atoms ( $\mathrm{p}$-doping) or $\mathrm{N}$ atoms ( $\mathrm{n}$-doping) are used. However, these substitutional dopants lead to resonant scattering effects ${ }^{118}$ that strongly depend on the distribution of the dopants and on the geometry of the graphene ribbons.

Magnetic Properties. Magnetism in pure carbon systems has recently been the subject of intense experimental and theoretical research (for an overview, see ref 119 and references therein). The driving force behind these studies was not only to create technologically important, light, nonmetallic magnets with a Curie point well above room temperature, but also to understand a fundamental problem: the origin of magnetism in a system which traditionally has been thought to show diamagnetic behavior only. In addition to polymerized fullerenes, ${ }^{120}$ nanotubes, ${ }^{121}$ graphite, ${ }^{122}$ and nanodiamonds, ${ }^{123}$ magnetism was recently reported for graphene produced from graphene oxide. ${ }^{124}$ On the basis of calculations, the observed magnetic behavior in all these systems was explained in terms of defects in the graphitic network (either native or produced by ion irradiation) such as under-coordinated carbon atoms, for example, vacancies, ${ }^{125}$ interstitials, ${ }^{126}$ carbon adatoms, ${ }^{47}$ and atoms at the edges of graphitic nanofragments with dangling bonds either passivated with hydrogen atoms or free. ${ }^{127}$ Such defects have local magnetic moments and may give rise to flat bands and eventually to the development of magnetic ordering. Magnetism may also originate from impurity atoms which are nonmagnetic by themselves (such as hydrogen or nitrogen), but because of the specific chemical environment give rise to local magnetic moments. With regard to magnetism in graphene, ${ }^{124}$ however, the samples were produced by annealing of graphene oxides and, surprisingly, the saturation magnetization did not correlate with the annealing temperature (a higher value for higher temperature). Moreover, no ferromagnetism was found at any temperature down to $2 \mathrm{~K}$ in very recent experiments ${ }^{128}$ on magnetization of graphene nanocrystals obtained by sonic exfoliation. No strong paramagnetism which has been expected due to the large amount of edge defects was reported either. Instead, above $50 \mathrm{~K}$ graphene showed strongly diamagnetic behavior, similar to graphite. In addition, a relatively weak but highly reproducible paramagnetic contribution was found at lower temperatures, which may be associated with the magnetic moments at the 
edges of small graphene crystallites. Overall, more studies are required to understand the origin of the observed magnetic effects in carbon systems and, first of all, graphene, as it has a simpler structure than other carbon nanomaterials. Here the controllable introduction of defects by irradiation followed by magnetic measurements could be particularly useful to explore the defect-mediated scenario.

Mechanical Properties. The influence of defects on the mechanical properties of graphene has not yet been studied experimentally. However, based on a large body of experimental and theoretical data for carbon nanotubes, ${ }^{129-132}$ one can expect that point defects, in particular vacancies, will decrease the Young's modulus and tensile strength of graphene samples. Conversely, an efficient reconstruction and healing of vacancy-type defects should minimize their detrimental effects. Line defects (dislocations) should be important for plastic deformation of graphene ribbons under tensile strain.

\section{COMPILATION OF DEFECT ENERGIES IN GRAPHENE}

Table 1 gives an overview of the calculated formation and migration energies of the most common types of point defects in graphene.

\section{CONCLUSIONS}

Although many outstanding properties of graphene are due to the inherently low concentration of defects, nanoengineering of graphene-based devices for dedicated functions needs the introduction of structural defects or impurities that allow us, like in conventional semiconductors, to achieve the desired functionality. The behavior of intrinsic zero- or one-dimensional defects in graphene such as vacancies or line defects is governed by the reconstruction of the graphenic lattice around defects, which is a unique property among all known materials. The high stability of the divacancy in its different configurations is of particular importance. The study of the influence of intrinsic defects on the electronic properties of graphene is still in its infancy, and experimental data relating defect concentration with the changes in electronic and optical characteristics are urgently needed. On the other hand, it is clear that extrinsic defects such as foreign atoms on different positions have a strong influence on the electron-electron interaction and thus charge distribution and the band structure of graphene. For example, transition metal atoms with $\mathrm{d}$ - and f-electrons embedded into the graphene lattice may give rise to many interesting phenomena including Kondo effect, magnetism, or charge- and spin-density waves. Doping with boron, nitrogen, or metals appears to become an important issue for the design of graphene-based devices in nanoelectronics.
Acknowledgment. Funding by the Agence Nationale de Recherche in France (NANOCONTACTS, NT09 507527) and the Academy of Finland through several projects is gratefully acknowledged. Our DFT calculations presented in this review were possible due to generous grants of computer time from the Finnish IT Center for Science. The authors are indebted to J. Meyer, R. Nieminen, K. Nordlund, L. Sun, J. A. Rodríguez-Manzo, O. Cretu, and C. Pham-Huu for fruitful collaboration.

\section{REFERENCES AND NOTES}

1. Kittel, C. Introduction to Solid State Physics; John Wiley \& Sons: New York, 2005.

2. Mermin, N. D. Crystalline Order in Two Dimensions. Phys. Rev. 1968, 176, 250-254.

3. Novoselov, K. S.; Geim, A. K.; Morozov, S. V.; Jiang, D.; Zhang, Y.; Dubonos, S. V.; Grigorieva, I. V.; Firsov, A. A. Electric Field Effect in Atomically Thin Carbon Films. Science 2004, 306, 666-669.

4. Geim, A. K. Graphene: Status and Prospects. Science 2009, $324,1530-1534$.

5. Castro Neto, A. H.; Guinea, F.; Peres, N. M. R.; Novoselov, K. S.; Geim, A. K. The Electronic Properties of Graphene. Rev. Mod. Phys. 2009, 81, 109-162.

6. Meyer, J. C.; Chuvilin, A.; Algara-Siller, G.; Biskupek, J.; Kaiser, U. Selective Sputtering and Atomic Resolution Imaging of Atomically Thin Boron Nitride Membranes. Nano Lett. 2009, 9, 2683-2689.

7. Bae, S.; Kim, H.; Lee, Y.; Xu, X.; Park, J.-S.; Zheng, Y.; Balakrishnan, J.; Lei, T.; Kim, H. R.; Song, Y. I.; et al. Roll-toRoll Production of 30-Inch Graphene Films for Transparent Electrodes. Nat. Nanotechnol. 2010, 5, 574-578.

8. Kim, K. S.; Zhao, Y.; Jang, H.; Lee, S. Y.; Kim, J. M.; Kim, K. S.; Ahn, J.-H.; Kim, P.; Choi, J.-Y.; Hong, B. H. Large-Scale Pattern Growth of Graphene Films for Stretchable Transparent Electrodes. Nature 2009, 457, 706-710.

9. Berger, C.; Song, Z.; Li, T.; Li, X.; Ogbazghi, A. Y.; Feng, R.; Dai, Z.; Marchenkov, A. N.; Conrad, E. H.; First, P.; et al. Ultrathin Epitaxial Graphite: 2D Electron Gas Properties and a Route Toward Graphene-Based Nanoelectronics. J. Phys. Chem. B 2004, 108, 19912.

10. Berger, C.; Song, Z.; Li, X.; Wu, X.; Brown, N.; Naud, C.; Mayou, D.; Li, T.; Hass, J.; Marchenkov, A. N.; et al. Electronic Confinement and Coherence in Patterned Epitaxial Graphene. Science 2006, 312, 1191-1196.

11. Novoselov, K. S.; Jiang, Z.; Zhang, Y.; Morozov, S. V.; Stormer, H. L.; Zeitler, U.; Maan, J. C.; Boebinger, G. S.; Kim, P.; Geim, A. K. Room-Temperature Quantum Hall Effect in Graphene. Science 2007, 315, 1379.

12. Hashimoto, A.; Suenaga, K.; Gloter, A.; Urita, K.; lijima, S. Direct Evidence for Atomic Defects in Graphene Layers. Nature 2004, 430, 870-873.

13. Gass, M. H.; Bangert, U.; Bleloch, A. L.; Wang, P.; Nair, R. R.; Geim, A. K. Free-Standing Graphene at Atomic Resolution. Nat. Nanotechnol. 2008, 3, 676-681.

14. Meyer, J. C.; Kisielowski, C.; Erni, R.; Rossell, M. D.; Crommie, M. F.; Zettl, A. Direct Imaging of Lattice Atoms and Topological Defects in Graphene Membranes. Nano Lett. 2008, 8, 3582-3586.

15. Warner, J. H.; Rümmeli, M. H.; Ge, L.; Gemming, T.; Montanari, B.; Harrison, N. M.; Büchner, B.; Briggs, G. A. D. Structural Transformations in Graphene Studied with High Spatial and Temporal Resolution. Nat. Nanotechnol. 2009, 4, 500-504.

16. Girit, Ç. O.; Meyer, J. C.; Erni, R.; Rossell, M. D.; Kisielowski, C.; Yang, L.; Park, C.-H.; Crommie, M. F.; Cohen, M. L.; Louie, S. G.; et al. Graphene at the Edge: Stability and Dynamics. Science 2009, 323, 1705-1708.

17. Ugeda, M. M.; Brihuega, I.; Guinea, F.; Gómez-Rodríguez, J. M. Missing Atom as a Source of Carbon Magnetism. Phys. Rev. Lett. 2010, 104, 096804.

18. Tapasztó, L.; Dobrik, G.; Nemes-Incze, P.; Vertesy, G.; Lambin, P.; Biró, L. P. Tuning the Electronic Structure of Graphene by Ion Irradiation. Phys. Rev. B 2008, 78, 233407.

19. Lu, A. J.; Pan, B. C. Nature of Single Vacancy in Achiral Carbon Nanotubes. Phys. Rev. Lett. 2004, 92, 105504. 
20. Kotakoski, J.; Krasheninnikov, A. V.; Nordlund, K. Energetics, Structure and Long-Range Interaction of Vacancy Defects on Carbon Nanotubes: Atomistic Simulations. Phys. Rev. B 2006, 74, 245420.

21. Miyamoto, Y.; Berber, S.; Yoon, M.; Rubio, A.; Tománek, D. Can Photo Excitations Heal Defects in Carbon Nanotubes. Chem. Phys. Lett. 2004, 392, 209-213.

22. Krasheninnikov, A. V.; Lehtinen, P. O.; Foster, A. S.; Nieminen, R. M. Bending the Rules: Contrasting Vacancy Energetics and Migration in Graphite and Carbon Nanotubes. Chem. Phys. Lett. 2006, 418, 132-136.

23. Rossato, J.; Baierle, R. J.; Fazzio, A.; Mota, R. Vacancy Formation Process in Carbon Nanotubes: First-Principles Approach. Nano Lett. 2005, 5, 197-200.

24. El-Barbary, A. A.; Telling, R. H.; Ewels, C. P.; Heggie, M. I.; Briddon, P. R. Structure and Energetics of the Vacancy in Graphite. Phys. Rev. B 2003, 68, 144107.

25. Ewels, C. P.; Telling, R. H.; El-Barbary, A. A.; Heggie, M. I. Briddon, P. R. Metastable Frenkel Pair Defect in Graphite: Source of Wigner Energy. Phys. Rev. Lett. 2003, 91, 025505.

26. Krasheninnikov, A. V.; Elesin, V. F. The Effect of Interstitial Clusters and Vacancies on the STM Image of Graphite. Surf. Sci. 2000, 519, 454-456.

27. Thrower, P. A.; Mayer, R. M. Point Defects and SelfDiffusion in Graphite. Phys. Stat. Sol. (a) 1978, 47, 11-36.

28. Kelly, B. T. Physics of Graphite; Applied Science: London, 1981.

29. Telling, R. H.; Heggie, M. I. Radiation Defects in Graphite. Philos. Mag. 2007, 87, 4797-4846.

30. Stone, A. J.; Wales, D. J. Theoretical Studies of Icosahedral $\mathrm{C}_{60}$ and Some Related Species. Chem. Phys. Lett. 1986, 128, 501-503.

31. Li, L.; Reich, S.; Robertson, J. Defect Energies of Graphite: Density-Functional Calculations. Phys. Rev. B 2005, 72, 184109.

32. Ma, J.; Alfé, D.; Michaelides, A.; Wang, E. Stone-Wales Defects in Graphene and Other Planar $\mathrm{sp}^{2}$-Bonded Materials. Phys. Rev. B 2009, 80, 033407.

33. Yazyev, O. V.; Tavernelli, l.; Rothlisberger, U.; Helm, L. Early Stages of Radiation Damage in Graphite and Carbon Nanostructures: A First-Principles Molecular Dynamics Study. Phys. Rev. B 2007, 75, 115418.

34. Kotakoski, J.; Jin, C. H.; Lehtinen, O.; Suenaga, K Krasheninnikov, A. V. Electron Knock-on Damage in Hexagonal Boron Nitride Monolayers. Phys. Rev. B 2010, 81,113404

35. Banhart, F. Irradiation Effects in Carbon Nanostructures. Rep. Prog. Phys. 1999, 62, 1181-1221.

36. Smith, B. W.; Luzzi, D. W. Electron Irradiation Effects in Single Wall Carbon Nanotubes. J. Appl. Phys. 2001, 90, 3509-3515.

37. McKinley, W. A.; Feshbach, H. The Coulomb Scattering of Relativistic Electrons by Nuclei. Phys. Rev. 1948, 74, 1759-1763.

38. Fukata, N.; Kasuya, A.; Suezawa, M. Formation Energy of Vacancy in Silicon Determined by a New Quenching Method. Phys. B 2001, 308-310, 1125-1128.

39. Maier, K.; Peo, M.; Saile, B.; Schaefer, H. E.; Seeger, A. HighTemperature Positron Annihilation and Vacancy Formation in Refractory Metals. Philos. Mag. A 1979, 40, 701-728.

40. Banhart, F.; Füller, T.; Redlich, Ph.; Ajayan, P. M. The Formation, Annealing, and Self-Compression of Carbon Onions under Electron Irradiation. Chem. Phys. Lett. 1997 269, 349-355.

41. Urita, K.; Suenaga, K.; Sugai, T.; Shinohara, H.; lijima, S. In Situ Observation of Thermal Relaxation of InterstitialVacancy Pair Defects in a Graphite Gap. Phys. Rev. Lett. 2005, 94, 155502.

42. Banhart, F.; Li, J. X.; Krasheninnikov, A. V. Carbon Nanotubes under Electron Irradiation: Stability of the Tubes and their Action as Pipes for Atom Transport. Phys. Rev. B 2005, 71, 241408(R)

43. Lee, G.-D.; Wang, C. Z.; Yoon, E.; Hwang, N.-M.; Kim, D.-Y.;
Ho, K. M. Diffusion, Coalescence, and Reconstruction of Vacancy Defects in Graphene Layers. Phys. Rev. Lett. 2005, 95, 205501.

44. Terrones, H.; Terrones, M.; Hernández, E.; Grobert, N.; Charlier, J.-C.; Ajayan, P. M. New Metallic Allotropes of Planar and Tubular Carbon. Phys. Rev. Lett. 2000, 84, 17161719.

45. Jeong, B. W.; Ihm, J.; Lee, G.-D. Stability of Dislocation Defect with Two Pentagon-Heptagon Pairs in Graphene. Phys. Rev. B 2008, 78, 165403.

46. Lee, Y. H.; Kim, S. G.; Tomanek, D. Catalytic Growth of Single-Wall Carbon Nanotubes: An ab Initio Study. Phys. Rev. Lett. 1997, 78, 2393-2396.

47. Lehtinen, P. O.; Foster, A. S.; Ayuela, A.; Krasheninnikov, A. V.; Nordlund, K.; R. M; Nieminen, R. M. Magnetic Properties and Diffusion of Adatoms on a Graphene Sheet. Phys. Rev. Lett. 2003, 91, 017202.

48. Tsetseris, L.; Pantelides, S. T. Adatom Complexes and SelfHealing Mechanisms on Graphene and Single-Wall Carbon Nanotubes. Carbon 2009, 47, 901-908.

49. Ma, Y. Simulation of Interstitial Diffusion in Graphite. Phys. Rev. B 2007, 76, 075419.

50. Lusk, M. T.; Car, L. D. Nanoengineering Defect Structures on Graphene. Phys. Rev. Lett. 2008, 100, 175503.

51. Lusk, M. T.; Wu, D. T.; Car, L. D. Graphene Nanoengineering and the Inverse Stone-Thrower-Wales Defect. Phys. Rev. B 2010, 81, 155444.

52. Banhart, F. Interactions between Metals and Carbon Nanotubes: At the Interface between Old and New Materials. Nanoscale 2009, 1, 201-213.

53. Gan, Y.; Sun, L.; Banhart, F. One- and Two-Dimensional Diffusion of Metal Atoms in Graphene. Small 2008, 4, 587591.

54. Rodríguez-Manzo, J. A.; Cretu, O.; Banhart, F. Trapping of Metal Atoms in Vacancies of Carbon Nanotubes and Graphene. ACS Nano 2010, 4, 3422-3428.

55. Cretu, O.; Krasheninnikov, A. V.; Rodríguez-Manzo, J. A.; Sun, L.; Nieminen, R.; Banhart, F. Migration and Localization of Metal Atoms on Graphene. Phys. Rev. Lett. 2010, 105, 196102.

56. Anton, R.; Schneidereit, I. In Situ TEM Investigations of Dendritic Growth of Au Particles on HOPG. Phys. Rev. $B$ 1998, 58, 13874-13881.

57. Kong, K.; Choi, Y.; Ryu, B.-H.; Lee, J.-O.; Chang, H. Investigation of Metal/Carbon-Related Materials for Fuel Cell Applications by Electronic Structure Calculations. Mater. Sci. Eng., C 2006, 26, 1207-1210.

58. Chan, K. T.; Neaton, J. B.; Cohen, M. L. First-Principles Study of Metal Adatom Adsorption on Graphene. Phys. Rev. B 2008, 77, 235430

59. Sevinçli, H.; Topsakal, M.; Durgun, E.; Ciraci, S. Electronic and Magnetic Properties of 3d Transition-Metal Atom Adsorbed Graphene and Graphene Nanoribbons. Phys. Rev. B 2008, 77, 195434.

60. Krasheninnikov, A. V.; Lehtinen, P. O.; Foster, A. S.; Pyykkö, P.; Nieminen, R. M. Embedding Transition-Metal Atoms in Graphene: Structure, Bonding, and Magnetism. Phys. Rev. Lett. 2009, 102, 126807.

61. Wehling, T. O.; Balatsky, A. V.; Katsnelson, M. I. Lichtenstein, A. I.; Rosch, A. Orbitally Controlled Kondo Effect of Co Adatoms on Graphene. Phys. Rev. B 2010, 81, 115427

62. Schmalz, T. G.; Seitz, W. A.; Klein, D. J.; Hite, G. E. Elemental Carbon Cages. J. Am. Chem. Soc. 1988, 110, 1113-1127.

63. Tan, Y.-Z.; Xie, S.-Y.; Huang, R.-B.; Zheng, L.-S. The Stabilization of Fused-Pentagon Fullerene Molecules. Nat. Chem. 2009, 1, 450-460.

64. Nemec, N.; Tománek, D.; Cuniberti, G. Contact Dependence of Carrier Injection in Carbon Nanotubes: An Ab Initio Study. Phys. Rev. Lett. 2006, 96, 076802.

65. Ci, L.; Song, L.; Jin, C.; Jariwala, D.; Wu, D.; Li, Y.; Srivastava, A.; Wang, Z. F.; Storr, K.; Balicas, L.; et al. Atomic Layers of Hybridized Boron Nitride and Graphene Domains. Nat. Mater. 2010, 9, 430-435 
66. Boukhvalov, D. W.; Katsnelson, M. I. Chemical Functionalization of Graphene with Defects. Nano Lett. 2008, 8, 4373-4379.

67. Nevidomskyy, A. H.; Csányi, G.; Payne, M. C. Chemically Active Substitutional Nitrogen Impurity in Carbon Nanotubes. Phys. Rev. Lett. 2003, 91, 105502.

68. Malola, S.; Häkkinen, H.; Koskinen, P. Gold in Graphene: InPlane Adsorption and Diffusion. Appl. Phys. Lett. 2009, 94, 043106.

69. Santos, E. J. G.; Ayuela, A.; Sánchez-Portal, D. FirstPrinciples Study of Substitutional Metal Impurities in Graphene: Structural, Electronic and Magnetic Properties. New J. Phys. 2010, 12, 053012.

70. Kroto, H. W.; Heath, J. R.; O'Brien, S. C.; Curl, R. F.; Smalley, R. E. C60: Buckminsterfullerene. Nature 1985, 318, $162-163$.

71. Coraux, J.; N'Diaye, A. T.; Busse, C.; Michely, T. Structural Coherency of Graphene on $\operatorname{Ir}(111)$. Nano Lett. 2008, 8, 565-570.

72. Červenka, J.; Katsnelson, M. I.; Flipse, C. F. J. RoomTemperature Ferromagnetism in Graphite Driven by TwoDimensional Networks of Point Defects. Nat. Phys. 2009, 5, 840-844.

73. Lahiri, J.; Lin, Y.; Bozkurt, P.; Oleynik, I. I.; Batzill, M. An Extended Defect in Graphene as a Metallic Wire. Nat. Nanotechnol. 2010, 5, 326-329.

74. Yazyev, O. V.; Louie, S. G. Topological Defects in Graphene: Dislocations and Grain Boundaries. Phys. Rev. B 2010, 81, 195420.

75. Malola, S.; Häkkinen, H.; Koskinen, P. Structural, Chemical, and Dynamical Trends in Graphene Grain Boundaries. Phys. Rev. B 2010, 81, 165447.

76. Koskinen, P.; Malola, S.; Häkkinen, H. Self-Passivating Edge Reconstructions of Graphene. Phys. Rev. Lett. 2008, 101, 115502.

77. Koskinen, P.; Malola, S.; Häkkinen, H. Evidence for Graphene Edges Beyond Zigzag and Armchair. Phys. Rev. B 2009, 80, 073401.

78. Chuvilin, A.; Meyer, J. C.; Algara-Siller, G.; Kaiser, U. From Graphene Constrictions to Single Carbon Chains. New J. Phys. 2009, 11, 083019.

79. Ohta, T.; Bostwick, A.; Seyller, T.; Horn, K.; Rotenberg, E. Controlling the Electronic Structure of Bilayer Graphene. Science 2006, 313, 951-954.

80. Xia, F.; Farmer, D. B.; Lin, Y.; Avouris, Ph. Graphene FieldEffect Transistors with High On/Off Current Ratio and Large Transport Band Gap at Room Temperature. Nano Lett. 2010, 10, 715-718.

81. Telling, R. H.; Ewels, C. P.; El Barbary, A. A.; Heggie, M. I. Wigner Defects Bridge the Graphite Gap. Nat. Mater. 2003, 2, 333-337.

82. Teobaldi, G.; Ohnishi, H.; Tanimura, K.; Shluger, A. L. The Effect of van der Waals Interactions on the Properties of Intrinsic Defects in Graphite. Carbon 2010, 48, 4145-4161.

83. Cruz-Silva, E.; Botello-Méndez, A. R.; Barnett, Z. M.; Jia, X.; Dresselhaus, M. S.; Terrones, H.; Terrones, M.; Sumpter, B. G.; Meunier, V. Controlling Edge Morphology in Graphene Layers Using Electron Irradiation: From Sharp Atomic Edges to Coalesced Layers Forming Loops. Phys. Rev. Lett. 2010, 105, 045501.

84. Kim, K. S.; Zhao, Y.; Jang, H.; Lee, S. Y.; Kim, J. M.; Kim, K. S.; Ahn, J.-H.; Kim, P.; Choi, J.-Y.; Hong, B. H. Large-Scale Pattern Growth of Graphene Films for Stretchable Transparent Electrodes. Nature 2009, 457, 706-710.

85. Krasheninnikov, A.; Banhart, F. Engineering of Nanostructured Carbon Materials with Electron or lon Beams. Nat. Mater. 2007, 6, 723-733.

86. Krasheninnikov, A. V.; Nordlund, K. lon and Electron Irradiation-Induced Effects in Nanostructured Materials. J. Appl. Phys. 2010, 107, 071301.

87. Rodríguez-Manzo, J. A.; Banhart, F. Creation of Individual Vacancies in Carbon Nanotubes by Using an Electron Beam of $1 \AA$ A Diameter. Nano Lett. 2009, 9, 2285-2289.
88. Lucchese, M. M.; Stavale, F.; Martins Ferreira, E. H.; Vilani, C.; Moutinho, M. V. O.; Capaz, R. B.; Achete, C. A.; Jorio, A Quantifying lon-Induced Defects and Raman Relaxation Length in Graphene. Carbon 2010, 48, 1592-1597.

89. Stolyarova, E.; Stolyarov, D.; Bolotin, K.; Ryu, S.; Liu, L.; Rim, K. T.; Klima, M.; Hybertsen, M.; Pogorelsky, I. Pavlishin et al. Observation of Graphene Bubbles and Effective Mass Transport under Graphene Films. Nano Lett. 2009, 9, 332337.

90. Chen, J.-H.; Cullen, W. G.; Jang, C.; Fuhrer, M. S.; Williams, E. D. Defect Scattering in Graphene. Phys. Rev. Lett. 2009, 102, 236805.

91. Compagnini, G.; Giannazzo, F.; Sonde, S.; Raineri, V.; Rimini, E. Ion Irradiation and Defect Formation in Single Layer Graphene. Carbon 2009, 47, 3201-3207.

92. Lehtinen, O.; Kotakoski, J.; Krasheninnikov, A. V.; Tolvanen, A.; Nordlund, K.; Keinonen, J. Effects of Ion Bombardment on a Two-Dimensional Target: Atomistic Simulations of Graphene Irradiation. Phys. Rev. B 2010, 81, 153401.

93. Lemme, M. C.; Bell, D. C.; Williams, J. R.; Stern, L. A.; Baugher, B. W. H.; Jarillo-Herrero, P.; Marcus, C. M. Etching of Graphene Devices with a Helium Ion Beam. ACS Nano 2009, 3, 2674-2676.

94. Zobelli, A.; Gloter, A.; Ewels, C. P.; Seifert, G.; Colliex, C. Electron Knock-on Cross Section of Carbon and Boron Nitride Nanotubes. Phys. Rev. B 2007, 75, 245402.

95. Bagri, A.; Mattevi, C.; Acik, M.; Chabal, Y. J.; Chhowalla, M.; Shenoy, V. B. Structural Evolution During the Reduction of Chemically Derived Graphene Oxide. Nat. Chem. 2010, 2, 581-587.

96. Balog, R.; Jørgensen, B.; Nilsson, L.; Andersen, M.; Rienks, E.; Bianchi, M.; Fanetti, M.; Lægsgaard, E.; Baraldi, A.; Lizzit, L. Bandgap Opening in Graphene Induced by Patterned Hydrogen Adsorption. Nat. Mater. 2010, 9, 315-319.

97. Cantele, G.; Lee, Y.-S.; Ninno, D.; Marzari, N. Spin Channels in Functionalized Graphene Nanoribbons. Nano Lett. 2009 9, 3425-3429.

98. Peng, X.; Ahuja, R. Symmetry Breaking Induced Bandgap in Epitaxial Graphene Layers on SiC. Nano Lett. 2008, 8, 4464-4468.

99. Duplock, E. J.; Scheffler, M.; Lindan, P. J. D. Hallmark of Perfect Graphene. Phys. Rev. Lett. 2004, 92, 225502.

100. Cortijo, A.; Vozmediano, M. A. H. Effects of Topological Defects and Local Curvature on the Electronic Properties of Planar Graphene. Nucl. Phys. B 2007, 763, 293-308.

101. Rutter, G. M.; Crain, J. N.; Guisinger, N. P.; Li, T.; First, P. N.; Stroscio, J. A. Scattering and Interference in Epitaxial Graphene. Science 2007, 317, 219-222.

102. Wehling, T. O.; Novoselov, K. S.; Morozov, S. V.; Vdovin, E. E.; Katsnelson, M. I.; Geim, A. K.; Lichtenstein, A. I. Molecular Doping of Graphene. Nano Lett. 2008, 8, 173-177.

103. Lu, Y. H.; Chen, W.; Feng, Y. P.; He, P. M. Tuning the Electronic Structure of Graphene by an Organic Molecule. J. Phys. Chem. B 2009, 113, 2-5.

104. McCreary, K. M.; Pi, K.; Swartz, A. G.; Han, W.; Bao, W.; Lau, C. N.; Guinea, F.; Katsnelson, M. I.; Kawakami, R. K. Effect of Cluster Formation on Graphene Mobility. Phys. Rev. B 2010, 81, 115453.

105. Pedersen, T. G.; Flindt, C.; Pedersen, J.; Mortensen, N. A.; Jauho, A.-P.; Pedersen, K. Graphene Antidot Lattices: Designed Defects and Spin Qubits. Phys. Rev. Lett. 2008, 100, 136804.

106. Yazyev, O. V.; Louie, S. G. Electronic Transport in Polycrystalline Graphene. Nat. Mater. 2010, 9, 806-809.

107. Appelhans, D. J.; Carr, L. D.; Lusk, M. T. Embedded Ribbons of Graphene Allotropes: An Extended Defect Perspective. New J. Phys. In press.

108. Areshkin, D. A.; Gunlycke, D.; White, C. T. Ballistic Transport in Graphene Nanostrips in the Presence of Disorder: Importance of Edge Effects. Nano Lett. 2007, 7, 204-210.

109. Li, T. C.; Lu, S.-P. Quantum Conductance of Graphene Nanoribbons with Edge Defects. Phys. Rev. B 2008, 77, 085408. 
110. Gorjizadeh, N.; Farajian, A. A.; Kawazoe, Y. The Effects of Defects on the Conductance of Graphene Nanoribbons. Nanotechnol. 2009, 20, 015201.

111. Deretzis, I.; Fiori, G.; lannaccone, G.; La Magna, A. Effects Due to Backscattering and Pseudogap Features in Graphene Nanoribbons with Single Vacancies. Phys. Rev. B 2010, 81, 085427.

112. Wehling, T. O.; Yuan, S.; Lichtenstein, A. I.; Geim, A. K.; Katsnelson, M. I. Resonant Scattering by Realistic Impurities in Graphene. Phys. Rev. Lett. 2010, 105, 056802.

113. Reich, S.; Maultzsch, J.; Thomsen, C.; Ordejón, P. TightBinding Description of Graphene. Phys. Rev. B 2002, 66, 035412.

114. Giovanetti, G.; Khomyakov, P. A.; Brocks, G.; Karpan, V. M.; van den Brink, J.; Kelly, P. J. Doping Graphene with Metal Contacts. Phys. Rev. Lett. 2008, 101, 026803.

115. Jung, N.; Kim, N.; Jockusch, S.; Turro, N. J.; Kim, P.; Brus, L. Charge Transfer Chemical Doping of Few Layer Graphenes: Charge Distribution and Band Gap Formation. Nano Lett. 2009, 9, 4133-4137.

116. Coletti, C.; Riedl, C.; Lee, D. S.; Krauss, B.; Patthey, L.; von Klitzing, K.; Smet, J. H.; Starke, U. Charge Neutrality and Band-Gap Tuning of Epitaxial Graphene on SiC by Molecular Doping. Phys. Rev. B 2010, 81, 235401.

117. Charlier, J.-C.; Blase, X.; Roche, S. Electronic and Transport Properties of Nanotubes. Rev. Mod. Phys. 2007, 79, 677-732.

118. Biel, B.; Blase, X.; Triozon, F.; Roche, S. Anomalous Doping Effects on Charge Transport in Graphene Nanoribbons. Phys. Rev. Lett. 2009, 102, 096803.

119. Yazyev, O. V. Emergence of Magnetism in Graphene Materials and Nanostructures. Rep. Prog. Phys. 2010, 73, 056501.

120. Kumar, A.; Avasthi, D. K.; Pivin, J. C.; Tripathi, A.; Singh, F. Ferromagnetism Induced by Heavy-lon Irradiation in Fullerene Films. Phys. Rev. B 2006, 74, 153409.

121. Friedman, A. L.; Chun, H.; Jung, Y. J.; Heiman, D.; Glaser, E. R.; Menon, L. Possible Room-Temperature Ferromagnetism in Hydrogenated Carbon Nanotubes. Phys. Rev. B 2010, 81, 115461.

122. Ramos, M. A.; Barzola-Quiquia, J.; Esquinazi, P.; MuñozMartin, A.; Climent-Font, A.; García-Hernández, M. Magnetic Properties of Graphite Irradiated with MeV Ions. Phys. Rev. B 2010, 81, 214404.

123. Talapatra, S.; Ganesan, P. G.; Kim, T.; Vajtai, R.; Huang, M.; Shima, M.; Ramanath, G.; Srivastava, D.; Deevi, S. C.; Ajayan, P. M. Irradiation-Induced Magnetism in Carbon Nanostructures. Phys. Rev. Lett. 2005, 95, 097201.

124. Wang, Y.; Huang, Y.; Song, Y.; Zhang, X.; Ma, Y.; Liang, J.; Chen, $Y$. Room-Temperature Ferromagnetism of Graphene. Nano Lett. 2009, 9, 220-224.

125. Andriotis, A. N.; Menon, M.; Sheetz, R. M.; Chernozatonskii, L. Magnetic Properties of $C_{60}$ Polymers. Phys. Rev. Lett. 2003, 90, 026801.

126. Yazyev, O. V. Magnetism in Disordered Graphene and Irradiated Graphite. Phys. Rev. Lett. 2008, 101, 037203.

127. Son, Y.; Cohen, M. L.; Louie, S. G. Half-Metallic Graphene Nanoribbons. Nature 2006, 444, 347-349.

128. Sepioni, M.; Rablen, S.; Nair, R. R.; Narayanan, J.;Tuna, F.; Winpenny, R.; Geim, A. K.; Grigorieva, I. V. Limits on Intrinsic Magnetism in Graphene. Phys. Rev. Lett. 2010, 105, 207205.

129. Sammalkorpi, M.; Krasheninnikov, A. V.; Kuronen, A.; Nordlund, K.; Kaski, K. Mechanical Properties of Carbon Nanotubes with Vacancies and Related Defects. Phys. Rev. B 2004, 70, 245416.

130. Mielke, S. L.; Troya, S.; Zhang, S.; Li, J.-L.; Xiao, S.; Car, R.; Ruoff, R. S.; Schatz, G. C.; Belytschko, T. The Role of Vacancy Defects and Holes in the Fracture of Carbon Nanotubes. Chem. Phys. Lett. 2004, 390, 413-420.

131. Ding, F.; Jiao, K.; Wu, M.; Yakobson, B. I. Pseudoclimb and Dislocation Dynamics in Superplastic Nanotubes. Phys. Rev. Lett. 2007, 98, 075503.
132. Uberuaga, B. P.; Stuart, S. J.; Voter, A. F. Parallel Replica Dynamics for Driven Systems: Derivation and Application to Strained Nanotubes. Phys. Rev. B 2007, 75, 014301. 